\title{
Acoustic and electromagnetic quasimodes in dispersed random media
}

\author{
Xiaodun Jing, ${ }^{*}$ Ping Sheng, and Minyao Zhou \\ Exxon Research and Engineering Company, Route 22 East, Annandale, New Jersey 08801
}

(Received 26 March 1992)

\begin{abstract}
A generalized coherent-potential-approximation (GCPA) approach is developed for the identification of quasimodes in dispersed random media and the calculation of their dispersion relations. Application of this approach to colloidal suspensions of solid spheres yields the prediction of two acoustic modes in excellent agreement with the observations of a recent Brillouin-scattering experiment [J. Liu, L. Ye, D. A. Weitz, and P. Sheng, Phys. Rev. Lett. 65, 2602 (1990)]. We show that whereas the high-frequency mode is associated with the solid-sphere antiresonances, the low-frequency mode arises from the coupling of the internal resonances between neighboring spheres. Our theory yields quantitative agreement with the measured dispersion relations with no adjustable parameters, and provides a theoretical explanation for the observed frequency gaps in the excitation spectra. Application of the GCPA to the electromagnetic wave in dispersed random media is also described.
\end{abstract}

PACS number(s): 82.70.Dd, 03.40.Kf, 62.65. $+\mathrm{k}, 68.45 . \mathrm{Nj}$

\section{INTRODUCTION}

Classical wave propagation and scattering is a topic extensively studied by both the physics and the engineering communities. It is the conventional wisdom that an isotropic, homogeneous, elastic solid has one longitudinal and two transverse modes, and a fluid has only one longitudinal-acoustic mode due to its lack of shear restoring force. For the fluid-solid composites, there are generally two types of behavior depending on the composite microstructure. For fluid-saturated porous media, where both the solid and the fluid phases form connected networks, Biot [1] has predicted the existence of two longitudinal modes, in addition to the two shear modes, where the fast longitudinal mode travels predominantly in the solid frame and the slow longitudinal mode mainly in the fluid. This prediction was indeed confirmed experimentally [2]. On the other hand, for dispersed random media where the solid particles form a discrete dispersion embedded in a liquid matrix, i.e., colloidal suspensions, it has been shown both theoretically and experimentally that at low frequencies there is only one acoustic mode $[3,4]$. As the frequency increases so that the wavelength becomes comparable with the scale of random inhomogeneities, the character of wave propagation is generally expected to change drastically due to the possibility of strong multiple scattering. For the solid-liquid system, James [5] first derived a formula for the scattering of an acoustic wave by a solid sphere immersed in liquid, which served as the basis for the multiple-scattering calculations to follow. Many different theoretical schemes, such as the $T$-matrix method $[6,7]$ of Waterman, coherentpotential approximation (CPA) [8], and the quasicrystalline approximation $[3,4,8-10]$, have been developed for the calculation of multiple-scattering effects. Wavelocalization phenomena [11] have also been predicted to result from multiple and resonant scatterings. However, despite extensive studies there has still been a lack of systematic understanding of wave-transport behavior in the intermediate-frequency regime. This situation is evidenced by the developments described below.

Recent Brillouin-scattering experiments [12] on colloidal suspensions, consisting of monodispersed polymethylmethacrylate (PMMA) spheres dispersed in oil, have yielded the surprising results that show at angular frequencies higher than $\pi c_{l} / d$, where $c_{l}$ is the acoustic wave speed in the liquid and $d$ is the diameter of the sphere, there exist two distinct longitudinal modes whose characteristics vary with the concentration of the solid spheres, whereas in the low-frequency regime there is indeed only one acoustic mode. These experimental results directly challenge our conventional understanding in two respects. First, how can there be more than one compressional mode in a colloidal suspension where the system has no overall shear modulus? Second, since the occurrence of the two modes coincides with the regime of strong resonant scattering, even the existence of propagating modes is a surprise and contrary to the conventional view that only diffusive transport exists in the strongscattering regime [11]. Also, the measured dispersion relations contain features, e.g., gaps in the dispersion relations, that are not immediately understandable.

The primary motivation of this work is to find an explanation for the above experimentally observed phenomena. The results of our study show, surprisingly, that the behavior of wave transport in the intermediate-frequency regime can be described by a simple approach that is based on the generalization of the well-known coherentpotential approximation (CPA). In particular, our generalized CPA condition offers a simple avenue for the identification of quasimodes and the calculation of their dispersion relations [13]. In what follows, we first present the physics of our results in Sec. II before delving into the model description and formulation in Sec. III, followed by the presentation and discussion of numerical results in Secs. IV and V. Our calculational effort can be separated into four stages. In the simplest stage, we do a onesphere calculation which, however, contains the impor- 
tant short-range correlation between the solid sphere and the enveloping liquid in a dispersed random medium. In the second stage we do a two-sphere calculation in which all the multiple scattering between the two spheres is treated exactly. In the third stage we extend the calculation to four spheres and ten spheres and treat the multiple scattering between these spheres exactly. In the fourth stage we carry out direct numerical simulations. The purpose of these layers of effort is to verify the physics of our results, e.g., the effect of short-range order among the spheres, the effect of fluid visciosity, etc. While our main results are simple, it has to be understood in the correct context that there are many side effects which may affect the main results to some degree. Section VI extends our approach to the electromagnetic case and shows that in a system of randomly dispersed metallic spheres there can also be multiple quasimodes.

\section{STATEMENT OF MAIN RESULTS}

In Fig. 1 we show the experimental result that is the primary motivation for this work. The colloidal sample consists of monodispersed PMMA spheres with diameter $d=370 \mathrm{~nm}$ suspended in transparent oil. The data were obtained by Brillouin scattering in which the angle between the incident and the scattered laser beams determines the wave vector $q$ of the acoustic excitation, and the amount of frequency shift from the Rayleigh peak determines the frequency of the excitation. These directly follow from the momentum and energy conservation of inelastic light scattering by acoustic phonons. All data are plotted in renormalized, dimensionless units. It should be noted that the value of $q d$ extends from much smaller than 1 to 10 , i.e., the wavelength of the acoustic excitations extends continuously from being very much larger than the sphere size (or average intersphere separation), to being very much smaller than that scale. While

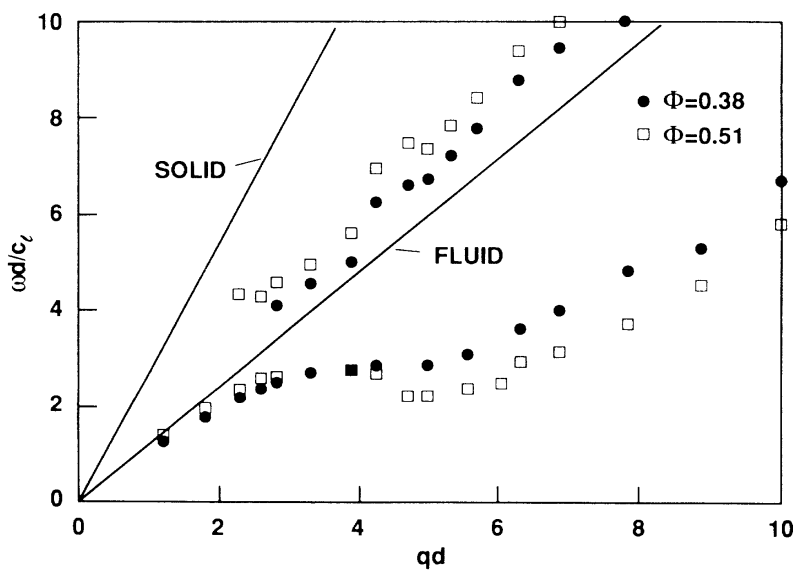

FIG. 1. Measured dispersion curves for PMMA colloidal suspensions at two concentrations. The diameter of the solid spheres is $d=370 \mathrm{~nm}$. The two solid straight lines are the dispersion curves for the longitudinal wave in the solid phase and the compressional wave in the liquid phase, as noted. not shown, each data point has an associated frequency width, representing its lifetime. The observed modes are therefore quasimodes.

At volume concentration $\Phi$ of solid spheres less than $20 \%$, there is only one mode whose dispersion relation deviates only slightly from that of the liquid. For $\Phi>20 \%$, there clearly appears two modes. Relative to the dispersion relations of the solid (that makes up the spheres) and the liquid, shown by the solid lines, the high-frequency mode has an intermediate velocity between those of the solid and the liquid, and it increases as $\Phi$ increases. These tendencies are in accord with general intuition. However, the high-frequency mode is clearly separated from the low-frequency mode by a gap. Moreover, there seem to exist smaller, higher-frequency gaps in the dispersion relation that are not immediately clear as to their origin(s).

The dispersion relation for the lower-frequency mode is seen to fall below that of the liquid, with a propagation velocity lower than that of the liquid. As $\Phi$ increases, the lower frequency mode actually slows down further, which is just opposite to the trend of the higherfrequency mode. Also, at higher sphere concentrations the dispersion relation is seen to develop a dip at $q d \approx 2 \pi$ that bears resemblance to the roton in liquid ${ }^{4} \mathrm{He}$. These features are the subject of our theoretical investigation.

In the context of the experimental results described above, the results of our study may be stated as follows [13]. First, the higher-frequency mode arises from the antiresonance condition for a single solid sphere immersed in liquid. That is, the mode appears in frequency ranges where there is a minimum in the cross section for acoustic-wave scattering from a solid sphere. The gaps in the dispersion relation thus correspond precisely to the frequency positions of the scattering cross-section peaks. The lower-frequency mode, on the other hand, arises from the coupling of the solid-sphere resonances between neighbors. This coupling is caused by the overlap of the decaying tails of the resonances on neighboring spheres in the liquid. One direct result of the coupling is the splitting of each resonance peak into two, with a minimum in between. Therefore the lower-frequency mode may be regarded as arising from the antiresonance condition of coupled neighboring spheres. Since the minimum here is generally at the frequency of a single sphere resonance, the frequency of the low-frequency mode thus corresponds to the gap position of the highfrequency mode.

The nature of the solid-sphere resonances offers an explanation for the low speed of the low-frequency mode. Just as for small metallic spheres the Mie resonances have their origin in the surface plasmon, here the solidsphere resonances are the manifestation of the Steoneley mode [14] in the spherical geometry. The Stoneley mode is known to be an interfacial mode bound to a flat liquidsolid interface with a wave velocity that is lower than either the liquid or the solid shear wave velocity. Thus as $\Phi$ increases, the low-frequency mode velocity decreases away from that of the liquid and approaches that of the Stoneley mode due to the increased solid-liquid interfacial area available to support its propagation. The dip in 
the dispersion relation of the low-frequency mode, on the other hand, is due to the short-range order of the spheres.

In view of the antiresonance condition as the unifying explanation for both the high- and low-frequency modes, a simple approach emerges for the identification and calculation of dispersion relations for the quasimodes. Its basic principle is simple and may be best understood as a generalization of the CPA condition [13]. For the CPA, if we let $G$ denote the exact Green's function (GF) for an acoustic wave in a random medium, then

$$
G=G_{0}+G_{0} T G_{0},
$$

where $G_{0}$ is the GF for a homogeneous effective medium and $T$ denotes the exact scattering operator, including all the multiple scattering among the spheres. By expressing $G_{0}=\left(p^{2}-q^{2}\right)^{-1}$, where $q=\omega / c_{m}, c_{m}$ being the effective-medium wave speed and $p$ the Fourier-transform variable that is conjugate to the spatial variable, the CPA condition for Eq. (2.1) is $\langle T\rangle=0$ through the adjustment of $q$, where the angular brackets denote configuration average. When that happens, $\langle G\rangle=G_{0}$, and $q$ is identified as the wave vector of the excitation. Since $\langle T\rangle \approx n t$ in the weak-scattering limit, where $t$ is the single-scatterer forward-scattering amplitude, $n$ the volume concentration of the scatterer, $\langle T\rangle=0$ is equivalent to $t=0$, which means the CPA condition is consistent with requiring the forward-scattering amplitude to vanish for a single scatterer embedded in an effective medium. In other words, when the scattering vanishes for a single scatterer, it also vanishes for the whole medium on average, and this is precisely the condition for the existence of a coherent mode.

For the generalized CPA condition, instead of requiring $\langle T\rangle=0$, we look for minima of $\langle T\rangle$. The fact that the scattering now does not vanish on average means that the excitation must be a quasimode. However, since at minima the scattering may still be weak, we may approximate $\langle G\rangle$ by

$$
\langle G\rangle \simeq \frac{1}{p^{2}-q^{2}-n t} .
$$

The minima of $\langle T\rangle$ may thus be identified by the maxima of density of state (DOS), evaluated as $-(1 / \pi) \operatorname{Im}\langle G\rangle$ under the condition of elastic scattering $(p=q)$, so that $\langle G\rangle \simeq-(n t)^{-1}$. The maxima of DOS therefore correspond directly to the minima in scattering, which, in turn, give the best condition for the existence of a quasimode since less scattering means the wave can coherently propagate over a longer distance. In this approach we also note that the minima in $\langle T\rangle$ are also the places where $\langle T\rangle \simeq n t$ is the best approximation. Therefore, the maxima of DOS are precisely the places where this approach is most accurate. In the following sections we give details and justifications for the results stated in this section.

\section{MODEL DESCRIPTION AND FORMULATION}

\section{A. Model description}

A colloidal suspension is characterized by the dispersive microgeometry, where each solid particle is individu- ally enveloped by liquid. To carry out our calculations, this strong short-range correlation between the solid and the liquid phases is taken into account in our one-sphere model by considering as the basic scattering unit a solid sphere of radius $a$ with a liquid coating of thickness $(b-a)$ as shown in Fig. 2(a). The volume fraction of the solid phase is given by $\Phi=a^{3} / b^{3}$. To calculate the effective macroscopic property of the system, this coated sphere is embedded in a homogeneous effective medium composed of similar units of coated spheres with the wave speed(s) $c_{m}$ to be determined by some self-consistent

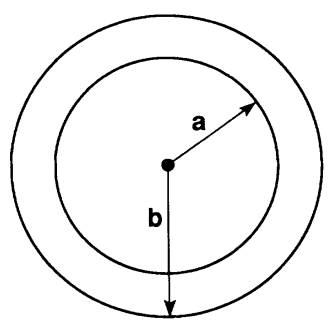

(a)

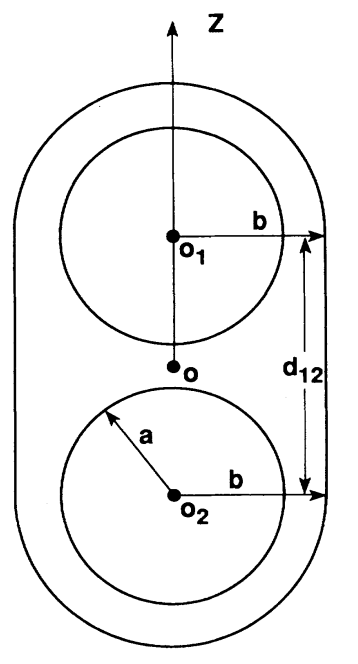

(b)

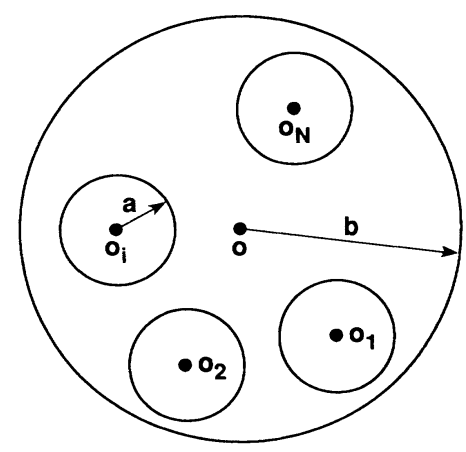

(c)

FIG. 2. Schematic sketches of the different scattering units in our calculation. (a) Single-coated sphere. (b) Scattering unit comprised of two spheres. (c) Spherical scattering unit comprised of $N$ spheres. 
condition. In the coherent-potential approximation [15], this condition is the requirement that the forwardscattering amplitude vanishes through the adjustment of $c_{m}$. Since macroscopically the system can only support compressional wave(s), we take the effective medium to be fluidlike. In this single-sphere model the largest error is incurred in treating the environment of each sphere as homogeneous. An improvement to the single coated sphere model is the case where there are $N$ spheres in the scattering unit, with the multiple scattering among all the spheres inside the scattering unit treated exactly. Different local environments of the spheres may be taken into account by the random configurations of the solid spheres in the scattering unit. The case for $N=2$ is shown in Fig. 2(b). In this case the scattering unit is in the form of a capsule, with radii $b$ at both ends and separation $d_{12}$ between the centers of the two spheres. The parameters $b$ and $d_{12}$ are related to the volume fraction $\Phi$ of the solid phase by $\Phi=8 a^{3} /\left(4 b^{3}+3 d_{12} b^{2}\right)$. In Fig. 2(c), the general case of four spheres in the scattering unit is shown where the surface of the scattering unit is spherical with radius $b$. Volume fraction of the solid phase is given by $\Phi=N a^{3} / b^{3}$. In Fig. 2(c), $O_{i}$ is the center position for the $i$ th solid sphere, $\mathbf{d}_{i}$ is the radial vector of the $i$ th sphere center relative to the origin $O ; \mathrm{d}_{i j}$ is the displacement vector from point $O_{i}$ to point $O_{j}$. In each of the models described above, we denote by region I that the solid phase, which has $N$ separate domains; by region II that of liquid phase inside the scattering unit; and by region III that which corresponds to the effective medium outside the scattering unit. The notations for the mass density, velocity(ies), and wave vectors in the three regions are, respectively, as follow. In region I, $\rho, c_{p}, c_{t}$, $k_{p}=\omega / c_{p}, k_{t}=\omega / c_{t}$; in region II, $\rho_{l}, c_{l}, k_{l}=\omega / c_{l}$; and in region III, $\rho_{m}, c_{m}, k_{m}=\omega / c_{m}$. Here $\rho_{m}=\Phi \rho+(1-\Phi) \rho_{l}$ is the average of mass density, and $c_{p}$ and $c_{t}$ are the compressional and shear wave speeds in the solid, respectively.

\section{B. General Green's-function formulation}

According to the model description above, our calculation has two major steps. The first step involves the calculation of the single scattering-unit Green's function. The second step is to evaluate the net effect of multiple scattering through an effective-medium approach. Let $G_{0}$ be the GF for the homogeneous effective medium. $G_{0}$ is a function of momentum $p$, angular frequency $\omega$, and the effective-medium speed. Let $G^{(s)}$ denote the exact GF for a single-scattering unit embedded in the homogeneous effective medium. The scattering unit may be represented by a potential operator $U_{j}^{(s)}[8,15]$,

$$
G^{(s)}=G_{0}+G_{0} U_{j}^{(s)} G^{(s)},
$$

where the subscript " $j$ " denotes that the scattering unit is located at $\mathbf{r}_{j}$. The superscript " $s$ " represents the operator for the single scattering unit.

Let $T^{(s)}$ denote the exact total scattering operator of the scattering unit which includes all the multiple scattering inside the unit as well as the scattering from the effective medium outside. $T^{(s)}$ is given by

$$
G^{(s)}=G_{0}+G_{0} T_{j}^{(s)} G_{0} .
$$

From Eq. (3.1) and Eq. (3.2) we have

$$
U_{j}^{(s)}=T_{j}^{(s)}\left(1+G_{0} T_{j}^{(s)}\right)^{-1} .
$$

For $N_{c}$ identical scattering units centered at $\mathbf{r}_{1}, \mathbf{r}_{2}, \ldots, \mathbf{r}_{N_{c}}$, the exact GF of the system, which is denoted by $G$, satisfies Eq. (3.1) but with a $U$ for the total system,

$$
G=G_{0}+G_{0} U G
$$

$G$ may be similarly written in the form

$$
G=G_{0}+G_{0} T G_{0}
$$

by defining a total-scattering operator $T$ for the system. When the scattering by a single unit is weak, one can express $U$ as

$$
U \simeq \sum_{j} U_{j}^{(s)}
$$

by neglecting multiple scattering between the units.

\section{Generalized CPA condition and the DOS}

It should be noted that in Eqs. (3.1)-(3.4) $G^{(s)}, G_{0}$, $T_{j}^{(s)}, U_{j}^{(s)}, T$, and $U$ are all functions of the effectivemedium wave vector $k_{m}=\omega / c_{m}$ and the frequency $\omega$. The CPA condition for Eq. (3.2) is $\left\langle T_{j}^{(s)}\right\rangle=0$ through the adjustment of an effective-medium parameter $c_{m}$ (or $k_{m}$ ). When this happens, it follows that $\langle T\rangle=0$ also, where $T$ is the total $T$ matrix for a system of $N_{c}$ scattering units, because $T=\Sigma_{j} T_{j}^{(s)}$ as the scattering becomes weak. Therefore, satisfaction of the CPA condition means $\langle G\rangle=G_{0}$, and $k_{m}$ is identified as the wave vector of the medium. In this work a generalized version of the CPA condition is used to identify the excitation mode(s) in a colloidal suspension. That is, instead of requiring $\left\langle T^{(s)}\right\rangle=0$, we wish to identify the $k_{m}$ value(s) at which $\left\langle T^{(s)}\right\rangle$ is at a local minimum for a wave of given frequency $\omega$. Since the scattering among the scattering units is expected to be weak under this condition, we have

$$
U_{j}^{(s)} \simeq T_{j}^{(s)} .
$$

From Eq. (3.4) the total GF may thus be written as

$$
G \simeq G_{0}+G_{0} \sum_{j} T_{j}^{(s)} G
$$

or

$$
\langle G\rangle \simeq\left(G_{0}^{-1}-n_{s} T^{(s)}\right)^{-1},
$$

where $n_{s}$ is the number density of the identical scattering units, and $n_{s} T^{(s)}$ is the self-energy to first order in scattering strength. The real part of $n_{s} T^{(s)}$ renormalizes the value of the wave vector of the excitation(s), and its imaginary part gives the mean free path, or lifetime, of the quasimode(s). The minimization of $T^{(s)}$ is noted to be not only for determining $q$, but also for justifying the weakscattering assumption so that the problem may be cast in the form of a single-scattering-unit calculation. 
With the condition of elastic scattering $\left|\mathbf{p}_{1}\right|=\left|\mathbf{p}_{2}\right|=q=k_{m}$, the DOS $\mathcal{D}$ is given by

$$
\mathcal{D}(q, \omega)=-\frac{1}{\pi} \operatorname{Im}\langle G\rangle=\frac{1}{\pi} \operatorname{Im} \frac{1}{n_{s} T^{(s)}} .
$$

It is clear from Eq. (3.8) that the maxima of the DOS, which is a function of frequency $\omega$ and the excitation wave vector $q$, correspond directly to the minima of scattering. The condition of the maximum of DOS directly gives the dispersion relation of $\omega$ vs $q$.

The task now is therefore the calculation of $T^{(s)}$. It is easy to show that the energy shell component of the scattering matrix $T^{(s)}$ is

$$
T_{\mathbf{q}, \mathbf{q}}^{(s)}=-4 \pi f(\mathbf{q}, \mathbf{q})
$$

where $f(\mathbf{q}, \mathbf{q})$ is the forward-scattering amplitude of the scattering unit embedded in the effective medium. By using Eq. (3.9), the DOS of Eq. (3.8) becomes

$$
\mathscr{D}(\omega, q)=-\frac{1}{4 \pi n_{s}} \operatorname{Im} \frac{1}{f(q)}
$$

where $f(q)=(1 / 4 \pi) \int f(\mathbf{q}, \mathbf{q}) d \Omega$ represents the angular average of the forward-scattering amplitude over all incident wave directions. This averaging is necessary due to the inherent randomness in the system.

\section{T matrix for a single-coated sphere}

To calculate the GF for a single-coated sphere embedded in an effective medium, we start from the elastic wave equation for each of the three homogeneous regions [see Fig. 2(a)]

$$
\frac{\partial^{2} \mathbf{u}}{\partial t^{2}}=c_{t}^{2}(\mathbf{r}) \nabla^{2} \mathbf{u}+\left[c_{p}^{2}(\mathbf{r})-c_{t}^{2}(\mathbf{r})\right] \nabla(\boldsymbol{\nabla} \cdot \mathbf{u}),
$$

where $\mathbf{u}$ denotes displacement, $c_{p}(\mathbf{r})$ and $c_{t}(\mathbf{r})$ are, respectively, the longitudinal and transverse wave velocities at r. For the liquid coating and the effective medium, $c_{t}=0$. The displacement $\mathbf{u}$ can generally be decomposed into a longitudinal part and a shear part, where each part can be derived from a scalar potential $\psi$ and a vector potential A, respectively,

$$
\mathbf{u}=\boldsymbol{\nabla} \psi+\boldsymbol{\nabla} \times \mathbf{A} \text {. }
$$

Because of the spherical symmetry of the problem, the vector $\mathbf{A}$ can be further expressed as $\mathbf{A}=\nabla \times(\mathbf{r} \xi)$. Both $\psi$ and $\xi$ must satisfy the scalar-wave equation

$$
\begin{aligned}
& \nabla^{2} \psi+\frac{\omega^{2}}{c_{p}^{2}(\mathbf{r})} \psi=0, \\
& \nabla^{2} \xi+\frac{\omega^{2}}{c_{t}^{2}(\mathbf{r})} \xi=0 .
\end{aligned}
$$

The mass-density variation, $\boldsymbol{\nabla} \cdot \mathbf{u}$, is directly proportional to $\psi$. Since in Brillouin scattering the light is coupled to thermal excitations through refractive-index changes induced by density variations, the relevant GF is that for measuring the $\psi$ response to a point scalar source. Therefore, we need to know the GF that satisfies the equation

$$
\left[\nabla^{2}+\omega^{2} / c_{p}^{2}(\mathbf{r})\right] G_{\psi}^{(s)}\left(\mathbf{r}, \mathbf{r}^{\prime}\right)=\delta\left(\mathbf{r}-\mathbf{r}^{\prime}\right),
$$

where $c_{p}(\mathbf{r})$ is the longitudinal wave velocity of the solid sphere in region $I$, compressional wave velocity $c_{l}$ in region II, and the effective-medium velocity $c_{m}$ in region III. For $\xi$, on the other hand, we have inside the solid sphere

$$
\left[\nabla^{2}+\omega^{2} / c_{t}^{2}\right] G_{\xi}^{(s)}=0,
$$

and $G_{\xi}^{(s)}=0$ in other regions. $G_{\psi}^{(s)}$ and $G_{\xi}^{(s)}$ are coupled by the boundary conditions at each interface, i.e., the continuity of normal displacement and normal stress at both $r=a$ and $r=b$, and vanishing tangential stress at the solid-liquid interface $r=a$. Here we have taken the liquid to be inviscid. The effect of visciosity is investigated by numerical simulations to be described in Sec. V. The GF $G_{\psi}^{(s)}\left(\mathbf{r}, \mathbf{r}^{\prime}\right)$ is obtained by solving three boundaryvalue problems where the source point $\mathbf{r}^{\prime}$ is located in each of the three regions. By Fourier transforming $G_{\psi}^{(s)}\left(\mathbf{r}, \mathbf{r}^{\prime}\right)$, we get $G_{\psi}^{(s)}\left(\mathbf{p}_{1}, \mathbf{p}_{2}, q, \omega\right)$, where the dependence on $q\left(=k_{m}=\omega / c_{m}\right)$ and frequency $\omega$ is explicitly noted. Since the GF can be expanded in angular-momentum space as

$$
G_{\psi}^{(s)}\left(\mathbf{r}, \mathbf{r}^{\prime}\right)=\sum_{l, m} g_{\psi l}^{(s)}\left(r, r^{\prime}\right) Y_{l, m}(\widehat{\mathbf{r}}) Y_{l, m}^{*}\left(\hat{\mathbf{r}}^{\prime}\right)
$$

where $Y_{l, m}(\hat{\mathbf{r}})$ is spherical harmonics, the $\wedge$ symbol denotes a unit vector and $g_{\psi_{l}}^{(s)}$ is the $l$ th component of the GF in angular space. One can get the expression of the exact scattering matrix for a single-coated sphere in momentum space by using Eq. (3.2),

$$
T_{\mathrm{p}_{1}, \mathrm{p}_{2}}^{(s)}=(4 \pi)^{2} \sum_{l, m} t_{l}^{(s)}\left(p_{1}, p_{2}\right) Y_{l, m}\left(\hat{\mathrm{p}}_{1}\right) Y_{l, m}^{*}\left(\hat{\mathbf{p}}_{2}\right) \text {. }
$$

The general expression for $t_{l}^{(s)}\left(p_{1}, p_{2}\right)$ is given is Appendix A. Under the case of elastic scattering $\left(p_{1}=p_{2}\right)$, the DOS depends only on $T_{\mathrm{q}, \mathrm{q}}^{(s)}$, which may be simply expressed [5] as

$$
T_{\mathrm{q}, \mathrm{q}}^{(s)}=\sum_{l, m} 4 \pi t_{l}^{(s)}(q, q)=i 4 \pi \sum_{l}(2 l+1) \bar{A}_{l},
$$

where $\bar{A}_{l}$ is given by Eq. (A.9) in Appendix A. By substituting Eq. (3.18) into Eq. (3.10), one then gets the DOS directly as a function of frequency $\omega$ and wave vector of the excitation $q=k_{m}=\omega / c_{m}$.

\section{E. Forward-scattering amplitude for a cluster of spheres}

In the last section a single-coated sphere is treated as the basic scattering unit. In that case, while the strong short-range correlation between the solid phase and the liquid phase is taken into account, the model incurs an error in treating the inhomogeneous surrounding of each solid sphere as a homogeneous effective medium. To improve the realism of our model, in this section we consider the more complex scattering unit composed of $N$ spheres [See Fig. 2(c).] Multiple scattering among the spheres inside the scattering unit is calculated exactly. In this case, it is difficult to get an analytical expression for the general scattering matrix $T_{\mathrm{p}_{1}, \mathrm{p}_{2}}^{(s)}$. But under the condition of elastic scattering, the DOS depends only on the 
diagonal term $T_{q, q}^{(s)}$, which is related to the forwardscattering amplitude by Eq. (3.9). Therefore, we only need to know the forward-scattering amplitude $f(\mathbf{q}, \mathbf{q})$ of the basic scattering unit. In the following, we present the derivation for the case of $N=2$ in some detail. The generalization to the case of $N$ spheres is given in Appendix B.

We assume the scattering unit with $N=2$ is in the form of a capsule, as shown in Fig. 2(b). The origin $O$ of the system is chosen as the midpoint of the centers of the two spheres at $O_{1}$ and $O_{2}$. The radial vectors from $O_{1}$ to $\mathrm{O}$ and from $\mathrm{O}_{2}$ to $\mathrm{O}$ are denoted by $\mathrm{d}_{1}$ and $\mathrm{d}_{2}$, respectively. The displacement vector from point $O_{1}$ to $O_{2}$ is denoted by $d_{12}=d_{2}-d_{1}$. Let the radial vectors of any point in space relative to $O, O_{1}$, and $O_{2}$ be denoted as $\mathrm{r}$, $\mathbf{r}_{1}$, and $\mathbf{r}_{2}$, respectively. Then

$$
\mathbf{r}_{1}=\mathbf{r}-\mathbf{d}_{1}, \quad \mathbf{r}_{2}=\mathbf{r}-\mathbf{d}_{2} .
$$

$$
\psi=\left\{\begin{array}{l}
e^{i \mathbf{k}_{m} \cdot \mathbf{r}}+\sum_{l, m} Q_{l m}^{(M)} h_{l}\left(k_{m} r\right) Y_{l m}(\hat{\mathbf{r}}) \quad(\text { region III) } \\
\sum_{i=1}^{2} \sum_{l, m} Q_{l m}^{(i)} h_{l}\left(k_{l} r_{i}\right) Y_{l m}\left(\hat{\mathbf{r}}_{i}\right)+\sum_{l, m} W_{l m} j_{l}\left(k_{l} r\right) Y_{l m}(\widehat{\mathbf{r}}) \quad(\text { region II }),
\end{array}\right.
$$

where $h_{l}$ is the Hankel function of the first kind [where the usual superscript (1) is ignored since in our paper only the Hankel function of the first kind is used]. $Q_{l m}^{(1)}, Q_{l m}^{(2)}$ are the expansion coefficients of the $(\operatorname{lm})$ th component of the scattered waves from the two spheres; $W_{l m}$ and $Q_{l m}^{(M)}$ correspond to the coefficients for the scattered waves from surface $S$ in regions II and III, respectively.

The displacement in region I can be generally represented by the three vector spherical-harmonic functions [16] $\mathbf{L}_{l m}, \mathbf{M}_{l m}, \mathbf{N}_{l m}$. Therefore, inside the $i$ th sphere the displacement may be expressed as

$\mathbf{u}^{(i)}=\sum_{l, m} a_{l m}^{(i)} \mathbf{L}_{l m}\left(\mathbf{r}_{i}\right)+b_{l m}^{(i)} \mathbf{M}_{l m}\left(\mathbf{r}_{i}\right)+c_{l m}^{(i)} \mathbf{N}_{l m}\left(\mathbf{r}_{i}\right)$,

where $a_{l m}^{(i)}, b_{l m}^{(i)}$, and $c_{l m}^{(i)}$ are the corresponding expansion coefficients, and $i=1,2$. At the surface of each solid sphere there are four boundary conditions; at the surface $S$ there are two boundary conditions. So altogether there are $4 \times 2+2=10$ boundary conditions, which exactly equals the number of expansion coefficients in Eqs. (3.21) and (3.22). The four boundary conditions at the surface
The separation of the two spheres $d_{12}$ may vary from $d_{12}=d=2 a$ to a certain maximum value which is determined by the volume fraction of the solid sphere, $4 d /\left(2 d+3 d_{12}\right)=\Phi$.

Consider a compressional plane wave in the effectivemedium incident on the cluster scattering unit with a scalar potential $\psi=e^{i \mathbf{k}_{m} \cdot \mathbf{r}}$, which can be expanded in terms of spherical waves as

$$
\psi=e^{i \mathbf{k}_{m} \cdot \mathbf{r}}=\sum_{l, m} 4 \pi i^{l} j_{l}\left(k_{m} r\right) Y_{l m}(\hat{\mathbf{r}}) Y_{l m}^{*}\left(\hat{\mathbf{k}}_{m}\right) .
$$

Here $j_{l}$ is the spherical Bessel function of the first kind, and $\hat{\mathbf{r}}$ and $\hat{\mathbf{k}}_{m}$ are unit vectors in the directions of $\mathbf{r}$ and $\mathbf{k}_{m}$, respectively. The field in region III is a combination of the incident field and scattered field from the surface $S$ of the scattering unit. The field in region II is a superpofrom the surface $S$. The expressions for these fields are sition of scattered fields from the two spheres and that of each solid sphere are the continuity of normal displacement and normal stress, and the vanishing of the tangential stresses. The two boundary conditions at $S$ are simply the continuity of normal displacement and normal stress. By fitting the four boundary conditions at each of the two spheres and eliminating the three coefficients $a_{l m}^{(i)}$, $b_{l m}^{(i)}$, and $c_{l m}^{(i)}$, the following two equations are obtained:

$$
\begin{aligned}
& Q_{l m}^{(1)}+A_{l} \sum_{l^{\prime}, m^{\prime}} \sigma_{l m, l^{\prime} m^{\prime}}\left(\mathbf{d}_{21}\right) Q_{l^{\prime} m^{\prime}}^{(2)} \\
& +A_{l} \sum_{l^{\prime}, m^{\prime}} s_{l m, l^{\prime} m^{\prime}}\left(\mathbf{d}_{1}\right) W_{l^{\prime} m^{\prime}}=0, \\
& Q_{l m}^{(2)}+A_{l} \sum_{l^{\prime}, m^{\prime}} \sigma_{l m, l^{\prime} m^{\prime}}\left(\mathbf{d}_{12}\right) Q_{l^{\prime} m^{\prime}}^{(1)} \\
& \quad+A_{l} \sum_{l^{\prime}, m^{\prime}} s_{l m, l^{\prime} m^{\prime}}\left(\mathbf{d}_{2}\right) W_{l^{\prime} m^{\prime}}=0,
\end{aligned}
$$

where $A_{l}$ is the expansion coefficient of the scattered wave for a single solid sphere, given by Eq. (A6), and $s_{l^{\prime} m^{\prime}, l m}, \sigma_{l^{\prime} m^{\prime}, l m}$ are the coefficients for the translation formula for the spherical Bessel and Hankel functions $[17,18]$, i.e., if $\mathbf{r}=\mathbf{R}+\mathbf{D}$, then

$$
h_{l}(k r) Y_{l, m}(\hat{\mathbf{r}})= \begin{cases}\sum_{l^{\prime}, m^{\prime}} h_{l^{\prime}}(k R) Y_{l^{\prime}, m^{\prime}}(\hat{\mathbf{R}}) s_{l^{\prime} m^{\prime}, l m}(\mathbf{D}), & |\mathbf{D}|<|\mathbf{R}| \\ \sum_{l^{\prime}, m^{\prime}} j_{l^{\prime}}(k R) Y_{l^{\prime}, m^{\prime}}(\hat{\mathbf{R}}) \sigma_{l^{\prime} m^{\prime}, l m}(\mathbf{D}), & |\mathbf{D}|>|\mathbf{R}| .\end{cases}
$$

The explicit expressions for $s_{l^{\prime} m^{\prime}, l m}$ and $\sigma_{l^{\prime} m^{\prime}, l m}$ are given in Appendix B. To fit the boundary conditions at $S$, it is noted that because the surface $S$ is not spherical, the expansion coefficients are obtained by carrying out a surface integration over $S$ for the two equations corresponding to the boundary conditions. The resulting equations are 


$$
\begin{gathered}
\frac{\rho_{l}}{k_{l}} \sum_{l, m} \int\left\{\sum_{i=1}^{2} Q_{l m}^{(i)} \sum_{l^{\prime}, m^{\prime}} s_{l^{\prime} m^{\prime}, l m}\left(-\mathbf{d}_{i}\right) h_{l^{\prime}}\left(k_{l} r\right) Y_{l^{\prime} m^{\prime}}(\hat{\mathbf{r}})+W_{l m} j_{l}\left(k_{l} r\right) Y_{l m}(\hat{\mathbf{r}})\right\} Y_{l^{\prime \prime} m^{\prime \prime}}^{*}(\hat{\mathbf{r}}) d S \\
=\left.\frac{\rho_{m}}{k_{m}} \sum_{l, m} \int\left[Q_{l m}^{(M)} h_{l}\left(k_{m} r\right) Y_{l m}(\hat{\mathbf{r}})+4 \pi i^{l} j_{l}\left(k_{m} r\right) Y_{l m}^{*}\left(\hat{\mathbf{k}}_{m}\right) Y_{l m}(\hat{\mathbf{r}})\right] Y_{l^{\prime \prime} m^{\prime \prime}}^{*}(\hat{\mathbf{r}}) d S\right|_{\mathbf{r} \text { on } S} \\
\frac{1}{k_{l}} \int \frac{\partial}{\partial n} \sum_{l, m}\left\{\sum_{i=1}^{2} Q_{l m}^{(i)} \sum_{l^{\prime}, m^{\prime}} s_{l^{\prime} m^{\prime}, l m}\left(-\mathbf{d}_{i}\right) h_{l^{\prime}}\left(k_{l} r\right) Y_{l^{\prime} m^{\prime}}(\hat{\mathbf{r}})+W_{l m} j_{l}\left(k_{l} r\right) Y_{l m}(\hat{\mathbf{r}})\right\} Y_{l^{\prime \prime} m^{\prime \prime}}^{*}(\hat{\mathbf{r}}) d S \\
=\left.\frac{1}{k_{m}} \int \frac{\partial}{\partial n}\left\{\sum_{l, m}\left[Q_{l m}^{(M)} h_{l}\left(k_{m} r\right) Y_{l m}(\hat{\mathbf{r}})+4 \pi i^{l} j_{l}\left(k_{m} r\right) Y_{l m}^{*}\left(\hat{\mathbf{k}}_{m}\right) Y_{l m}(\hat{\mathbf{r}})\right]\right\} Y_{l^{\prime \prime} m^{\prime \prime}}^{*}(\hat{\mathbf{r}}) d S\right|_{\mathrm{r} \text { on } S}
\end{gathered}
$$

There are two generic integrals in the above two equations that can be written as

$I_{l m, l^{\prime} m^{\prime}}^{(1)}(k)=\left.\int I^{(1)}(k r(\theta)) Y_{l m}(\hat{\mathbf{r}}) Y_{l^{\prime} m^{\prime}}^{*}(\hat{\mathbf{r}}) d S\right|_{\mathrm{r} \text { on } S}$,

$I_{l m, l^{\prime} m^{\prime}}^{(2)}(k)=\left.\int I^{(2)}(k r(\theta))\left[\frac{\partial}{\partial n} Y_{l m}(\hat{\mathbf{r}})\right] Y_{l^{\prime} m^{\prime}}^{*}(\hat{\mathbf{r}}) d S\right|_{\mathbf{r} \text { on } S}$

where $I^{(1)}(k r(\theta))$ and $I^{(2)}(k r(\theta))$ are functions of $k r$, where $r$ is itself a function of polar angle $\theta$. The resultant integrals, $I_{l m, l^{\prime} m^{\prime}}^{(1)}(k)$ and $I_{l m, l^{\prime} m^{\prime}}^{(2)}(k)$, are functions of the wave vector $k$ and angular indices $l, m$. Because the scattering unit has azimuthal symmetry, the $z$ component of the angular momentum $m$ is conserved during the scattering process. As a result, all matrices in Eqs. (3.23) and (3.25) are diagonal with respect to $m$. Thus the scattering for different $m$ can be calculated separately. It is easy to show that

$$
I_{l, m ; l^{\prime}, m^{\prime}}^{(1)}=\left\{\begin{array}{l}
0, \quad m^{\prime} \neq m \\
0, \quad l+l^{\prime}=\text { odd } \\
I_{l,-m ; l^{\prime},-m^{\prime}}(k), \text { otherwise }
\end{array}\right.
$$

$I_{l m, l^{\prime} m^{\prime}}^{(2)}(k)$ and the relevant matrices in Eq. (3.23) have the same properties as $I_{l m, l^{\prime} m^{\prime}}^{(1)}$. The procedure for obtaining the forward-scattering amplitude is therefore as follows. First, the variables $Q_{l m}^{(1)}, Q_{l m}^{(2)}, W_{l m}$, and $Q_{l m}^{(M)}$ are solved from Eqs. (3.23) and (3.25). The forward-scattering amplitude may be obtained from the field expression in region III,

$$
f\left(\omega, \mathbf{k}_{m}\right)=\frac{1}{k_{m}} \sum_{l, m} Q_{l m}^{(\boldsymbol{M})}(-i)^{l+1} \boldsymbol{Y}_{l m}\left(\hat{\mathbf{k}}_{m}\right),
$$

which can be formally written as

$$
f\left(\omega, \mathbf{k}_{m}\right)=\frac{4 \pi i}{k_{m}} \sum_{l, m} \sum_{l^{\prime}, m^{\prime}} \Pi_{l m, l^{\prime} m^{\prime}} Y_{l^{\prime} m^{\prime}}^{*}\left(\hat{\mathbf{k}}_{m}\right) Y_{l m}\left(\hat{\mathbf{k}}_{m}\right),
$$

where the matrix $\Pi_{l m, l^{\prime} m^{\prime}}$ depends on the angular frequency $\omega$, the magnitude of the wave vector $k_{m}$, and the separation of the two spheres inside the scattering unit. Because the system is random, the forward-scattering amplitude is averaged over all possible incident directions of $\mathbf{k}_{m}$,

$$
\bar{f}\left(\omega, k_{m}\right)=\frac{1}{4 \pi} \int f\left(\omega, \mathbf{k}_{m}\right) d \Omega_{\mathbf{k}_{m}}=\frac{i}{k_{m}} \sum_{l, m} \Pi_{l m, l m}
$$

Equation (3.30) is then averaged over separation $d_{12}$ by using the radial distribution function for the hard-sphere model [19]. Finally, the DOS is obtained from Eq. (3.10) as a function of frequency and the magnitude of the wave vector in the effective medium.

In the $N=2$ case the surface integration is necessary because the scattering unit is in the form of a capsule. When more spheres are considered, the scattering unit is taken to be spherical in shape. Then no surface integration is needed. The case for $N=10$ with a spherical surface $S$ is a straightforward generalization of the case for $N=2$. Its derivation is given in Appendix B.

\section{RESULTS AND DISCUSSION}

\section{A. Single-coated sphere results}

In Figs. 3(a) and 3(b) we plot, in color, the calculated DOS as a function of the dimensionless frequency $\omega d / c_{l}$ and dimensionless wave vector $q d$ for volume fraction $\Phi=0.38$ and $\Phi=0.51$, respectively, for the single-sphere model. The volume fraction $\Phi$ determines the liquidcoating thickness used in the calculation. The material parameters used are $c_{p}=2.7 \times 10^{5} \mathrm{~cm} / \mathrm{sec}$ and $c_{t}=1.1 \times 10^{5} \mathrm{~cm} / \mathrm{sec}$ for PMMA, $c_{l}=1.2 \times 10^{5} \mathrm{~cm} / \mathrm{sec}$ for the liquid, and $\rho \approx \rho_{l} \approx 1 \mathrm{~g} / \mathrm{cm}^{3}$. Two bands of ridges are clearly seen in red and yellow. They correspond remarkably well to the experimentally observed dispersion relations for the two acoustic modes, shown as black points with bars indicating the measured frequency width. It should be stressed that the calculation has no adjustable parameters. While not shown, our calculations also indicate that for volume fraction below $\Phi \approx 0.20$ the two dispersion relations merge and become indistinguishable from the liquid dispersion relation, in agreement with the experimental observation [12].

The black arrows on the sides indicate the frequency positions of the peaks in the scattering cross section for a single solid sphere immersed in liquid, shown in Fig. 4. They are the key to understanding the physical origin of the two modes. For the high-frequency mode, the positions of the arrows correspond directly to the gaps in the calculated dispersion relation. This correspondence sug- 
gests that the high-frequency mode results from the antiresonance of a single sphere, where the scattering is minimum. The fact that the minima are nonzero implies that the excitations are quasimodes with finite lifetimes. The first gap, corresponding to the first scattering peak in Fig. 4 , is clearly seen in the experimental results for both $\Phi=0.51$ and 0.38 . The second gap, and hint of a third, are also visible.
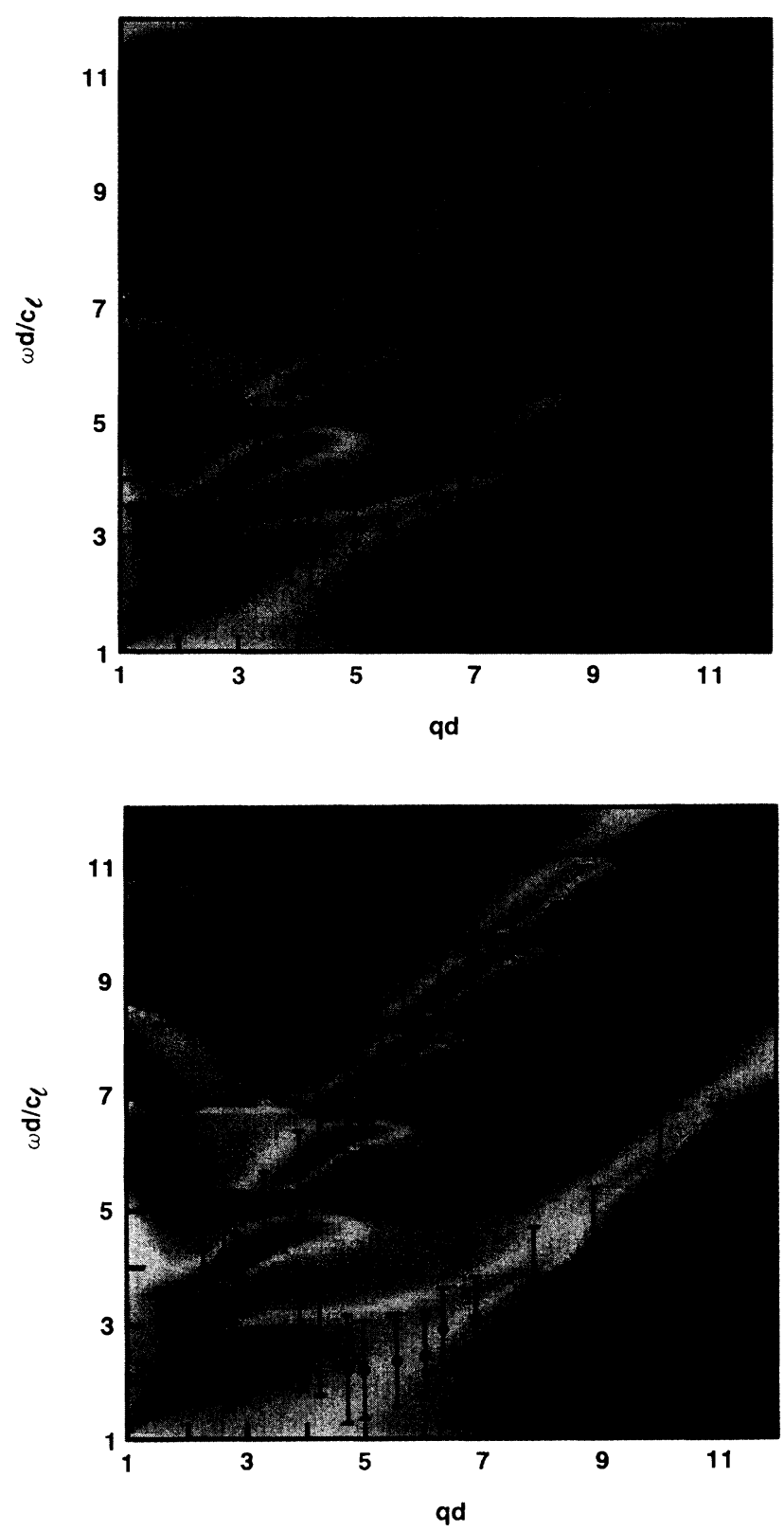

FIG. 3. The DOS plotted as a function of $\omega d / c_{l}$ and $q d$. The magnitude of the DOS is delineated by color, where high is indicated by red and low is indicated by blue. Yellow is intermediate. Experimental data are shown as solid circles. The bars indicate their frequency widths. The arrows on the sides indicate the frequency positions of the scattering cross-section peaks for a single sphere immersed in liquid, shown in Fig.4. (a) $\Phi=0.38$ and (b) $\Phi=0.51$.

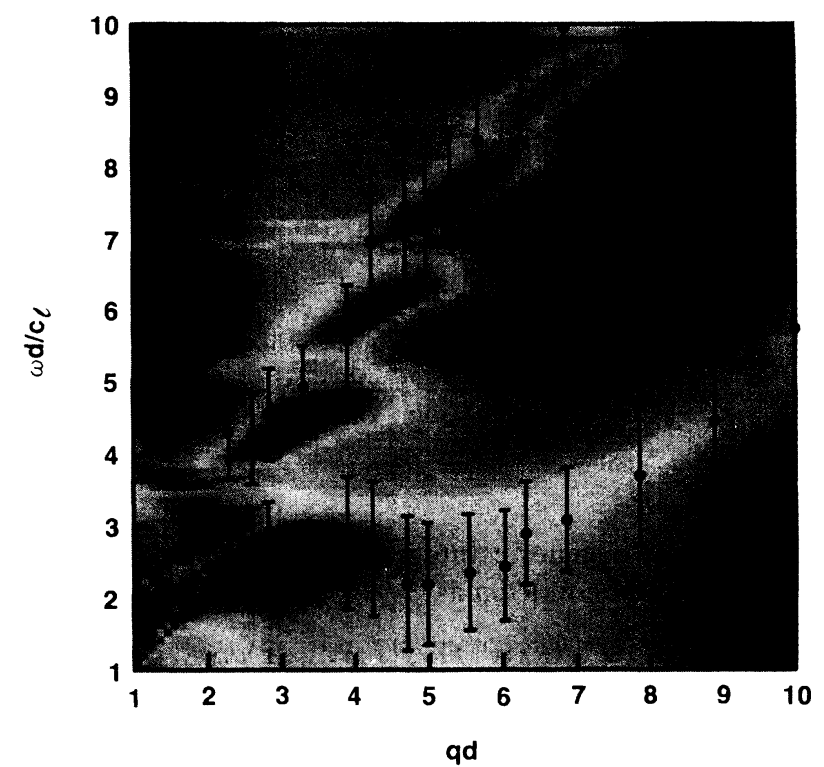

FIG. 11. The DOS for a two-sphere scattering unit embedded in an effective medium with $\Phi=0.51$. This figure should be compared with Fig. 3(b).

In Fig. 5 we analyze the partial-scattering cross section of different angular components for the first four main peaks. It is seen that the first peak in Fig. 4 corresponds to the $l=2$ partial wave, and the second peak to the $l=3$ partial wave. The third main peak is seen to consist of two small peaks. The different $l$ components of these two peaks exhibit only minor differences. As frequency increases, more $l$ components contribute to the same resonance peak. For each peak there exists a maximum $l_{\max } \approx \omega d / c_{l}$ beyond which the partial-scattering cross section decreases very rapidly and can be ignored.

In contrast to the behavior of the high-frequency mode, the peak DOS for the low-frequency mode corresponds to the single-sphere resonance frequency. In Fig. 3(a) the calculated gap in its dispersion relation is seen to correspond reasonably well with the experimentally observed one. The low-frequency mode is therefore the direct result of single-sphere resonances, and the peak of the DOS (or the minima of scattering) results from the interaction between neighboring spheres. That is, the resonances on neighboring spheres couple through their decaying portions in the liquid, causing a splitting of each peak into two, with a minimum in between. It is this minimum that is picked up as a peak in the DOS. For the case of a single-coated sphere, the interaction with the neighboring spheres may be represented by some particular value of wave impedance at the coating-effective medium interface. Since the effective medium $q$ is treated as a variable, we are thus able to pick up the scattering minimum by scanning its value. A more detailed verification of this physical picture is provided by the four-sphere calculation to be described in the next section.

As discussed above, both the high-frequency and the low-frequency modes depend on the existence of the single-sphere resonances. The nature of these resonances 


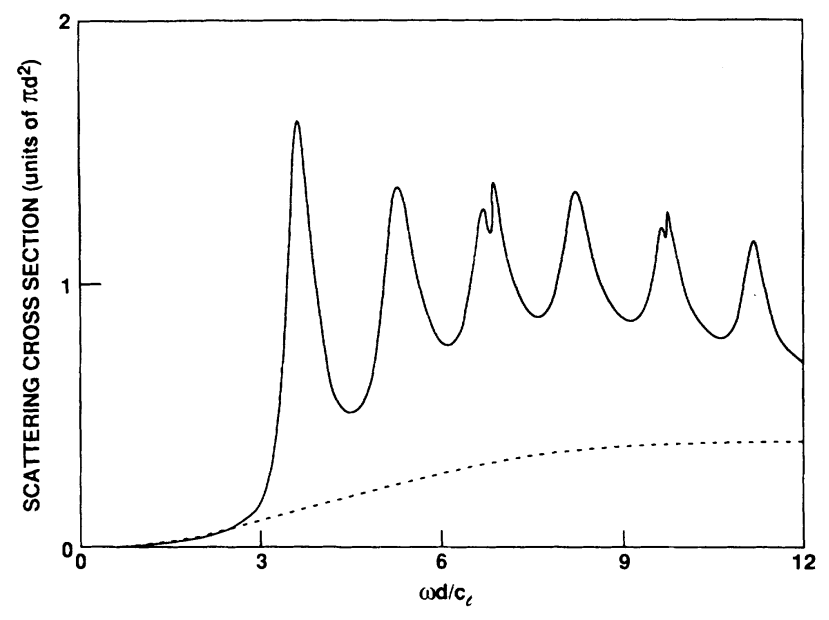

FIG. 4. Renormalized scattering cross section plotted as a function of frequency $\omega d / c_{l}$ for a solid sphere (solid curve) and a liquid sphere (dashed curve) immersed in fluid.

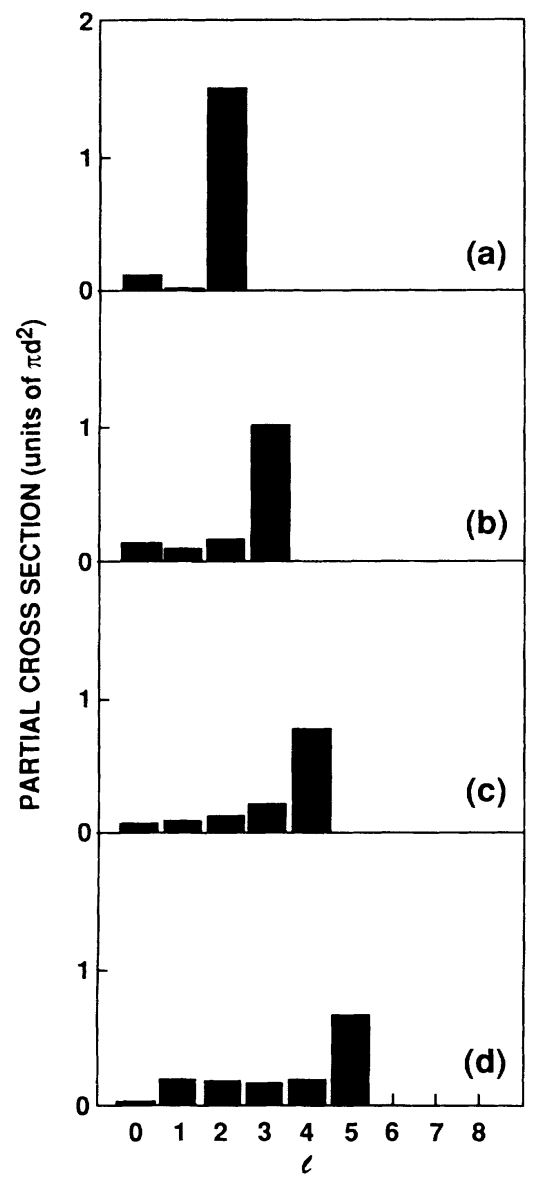

FIG. 5. Partial scattering cross section of different angular momentum $l$ for the first four main peaks in Fig. 4. (a) The first peak is predominantly $l=2$. (b) The second peak is predominantly $l=3$. (c) The third peak is split into two smaller peaks. What is shown is the lower frequency peak of the two. It has a large $l=4$ component, but the combined partial cross section from $l<4$ is non-negligible. The higher-frequency peak of the two has similar behavior. (d) Partial cross-section content of the fourth resonance.

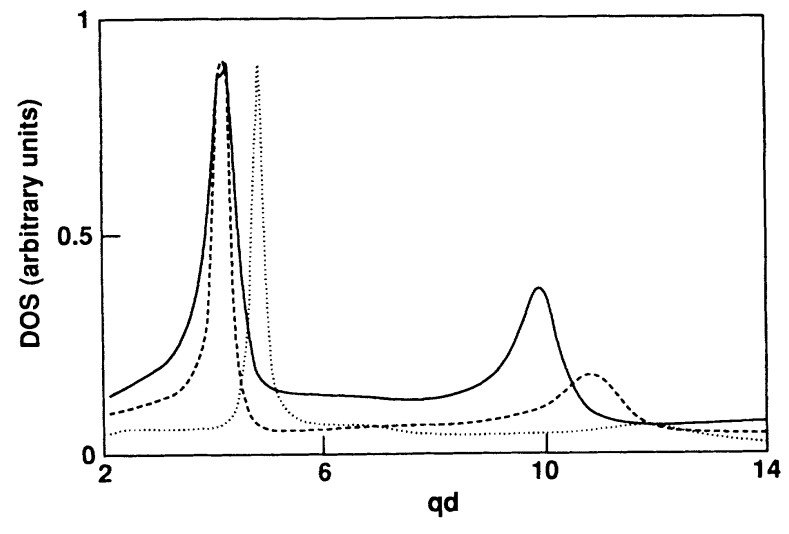

FIG. 6. The DOS plotted as a function of $q d$ for $\omega d / c_{l}=6$ and $\Phi=0.51$. The dashed and dotted lines correspond to cases where the shear modulus of the solid sphere is progressively decreased to 0.64 and 0.46 times of its physical value. Whereas the high-frequency mode is only slightly perturbed, the lowfrequency mode is seen to gradually disappear.

thus requires some examination. In Fig. 4 the scattering cross section is plotted as a function of frequency for a sphere immersed in liquid. Two case are shown. In one case the shear modulus $\mu$ of the sphere is set to zero (liquid sphere), and in the second case $\mu \neq 0$ (solid sphere). It is clearly evident that for the $\mu=0$ case the scattering peaks have disappeared, which illustrates the crucial role of a finite shear modulus. In fact, these resonances may be viewed as the solid-liquid interfacial "Stoneley mode" [14] (which also disappears if the shear modulus vanishes) specialized to the spherical geometry. They are the elastic analog to the "surface modes" of small metallic spheres [20] that may be viewed as an extension of the surface plasmon to the spherical geometry. If the frequency is high enough to ignore the sphere curvature, these resonant modes of a solid sphere may be approximated as standing waves at the surface of the sphere with about the same phase velocity as that of the Stoneley

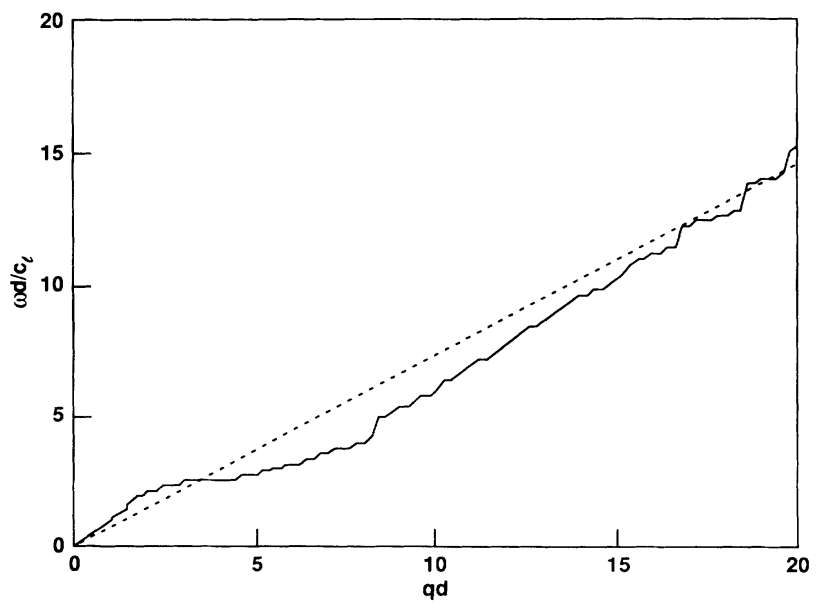

FIG. 7. The dispersion relation of the low-frequency mode calculated from the single-coated sphere model at $\Phi=0.51$ (solid line). The dashed line corresponds to the dispersion curve for the Stoneley wave at a flat interface. As frequency increases, the solid curve is seen to approach the dashed curve. 
wave at a flat solid-liquid interface $\left(c_{\text {Stoneley }}=0.86 \times 10^{5}\right.$ $\mathrm{cm} / \mathrm{sec}$ for the experimental parameters). The mode frequency should thus be $\omega d / c_{l}=2 m c_{\text {Stoneley }} / c_{l}$, where $m$ is an integer. On this basis, the separation of the resonant modes is expected to be $2 c_{\text {Stoneley }} / c_{l}=1.46$. The resonance peaks in Fig. 4 are seen to be about equally spaced with a separation of 1.48 , which is almost exactly the predicted value.

Since the high-frequency mode depends on the antiresonance condition whereas the low-frequency mode depends on the resonance condition (with interaction), they are expected to exhibit distinct behaviors as the shear modulus varies. Figure 6 demonstrates the effects of the shear modulus on the two modes. At a fixed frequency, the DOS is plotted as a function of the dimensionless wave vector $q d$. The two peaks correspond to the two modes. As the shear modulus is progressively decreased, the high-frequency mode (low $q d$ ) shows only minor shifts, but the low-frequency mode not only shifts to higher $q d$ but also diminishes in amplitude, indicating that the shear modulus is responsible for the strength of the low-frequency mode. In contrast, for the highfrequency mode the disappearance of the resonances only implies the disappearance of gaps in its dispersion relation. The physical picture that the low-frequency mode results from the coupling of the surface Stoneley wave is further supported by the fact that in both theory and ex- periment the speed of the low-frequency mode approaches that of the Stoneley wave at the large $q d$ limit, where the surface-curvature effect becomes negligible. In Fig. 7 the calculated frequency peak position of the lowfrequency mode is plotted as a function of $q d$. As $q d$ increases, its dispersion curve is seen to gradually approach that of the Stoneley wave on a flat solid-liquid interface, represented by the straight dashed line. Thus at the large $q d$ limit the low-frequency mode represents a standing wave at the sphere's surface, with no propagation between the spheres. However, as $q d$ decreases below 10 propagation between spheres becomes possible due to the tail coupling. Since the Stoneley-wave speed is below that of the liquid, the speed of the low-frequency mode thus decreases as $\Phi$ increases due to the increased interfacial area available to support its propagation.

\section{B. The scattering of two and four spheres immersed in liquid}

While the single-sphere case already contains the essential physics of the system, it cannot give details that are explicitly related to the interaction between two or more spheres. To further clarify this point, we have carried out the calculation of two spheres and four spheres immersed in liquid. In Fig. 8 the total-scattering cross sections, corresponding to three different incident angles
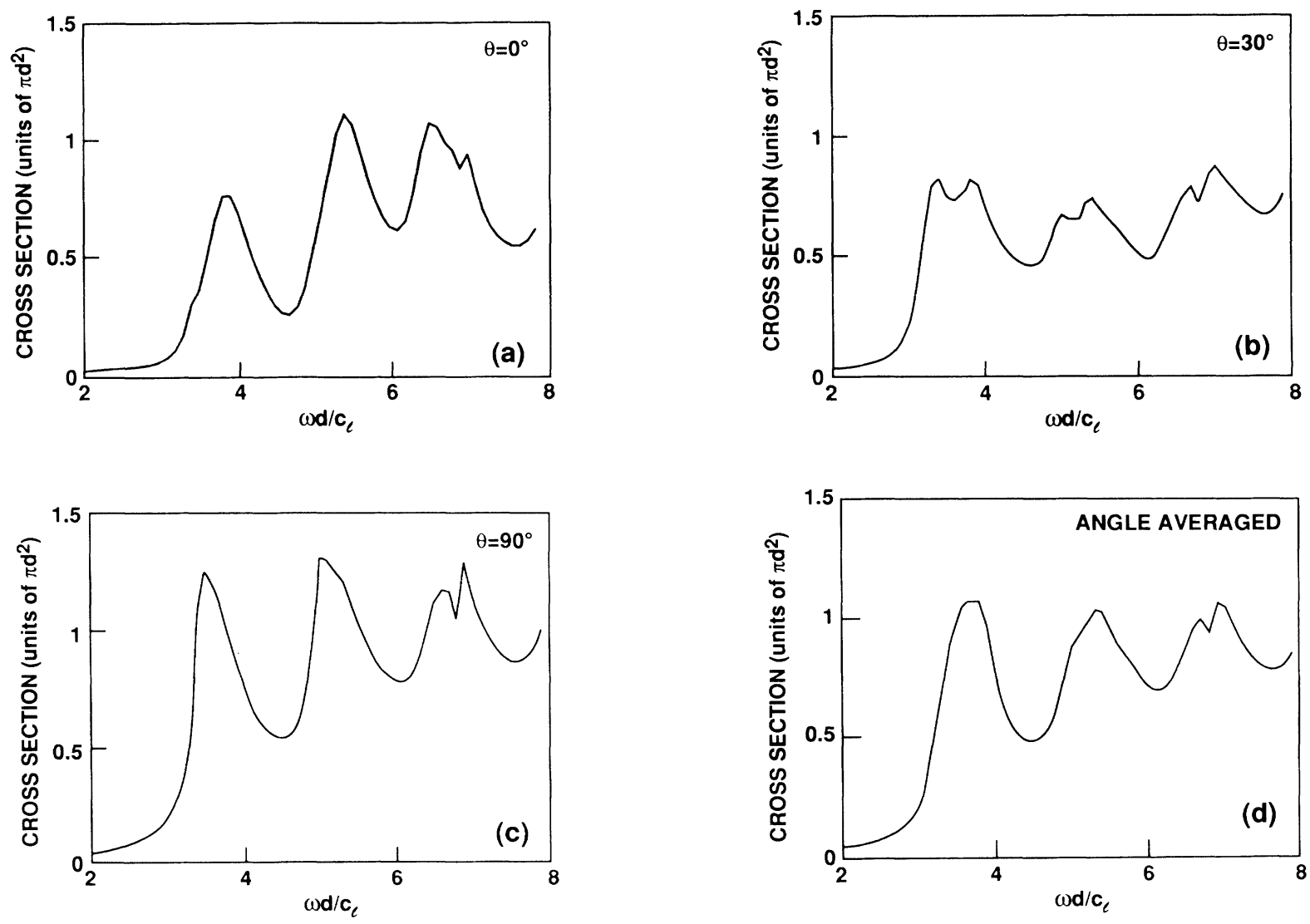

FIG. 8. Renormalized scattering cross section of two solid spheres immersed in liquid for three different incident angles: (a) $\theta=0^{\circ}$, (b) $\theta=30^{\circ}$, (c) $\theta=90^{\circ}$, and (d) the result of averaging over all angles. The angle $\theta$ is defined relative to the axis joining the centers of the two spheres. The cutoff angular momentum used in the calculation is $l_{0}=10$. 


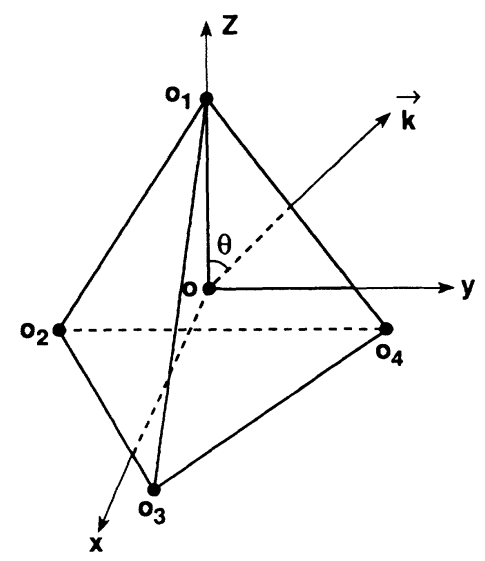

FIG. 9. Geometry of the centers of four spheres.

of $\theta=0^{\circ}, 30^{\circ}$, and $90^{\circ}$, are plotted as a function of the reduced frequency $\omega d / c_{l}$. Here the separation of the two spheres, $d_{12}$, is approximately equal to the diameter of the sphere $d$, and $\theta$ is defined relative to the axis joining the centers of the two spheres [the $z$ axis in Fig. 2(b)]. We found that when $\theta$ is around $30^{\circ}$, the interscattering between the two spheres can result in the splitting of each peak into two [Fig. 8(b)]: However, for the parallel $\left(\theta \approx 0^{\circ}\right)$ and the perpendicular incidences $\left(\theta \approx 90^{\circ}\right)$, the interscattering between the two spheres is apparently not strong enough to split the single-sphere scattering peak [see Figs. 8(a) and 8(c)].
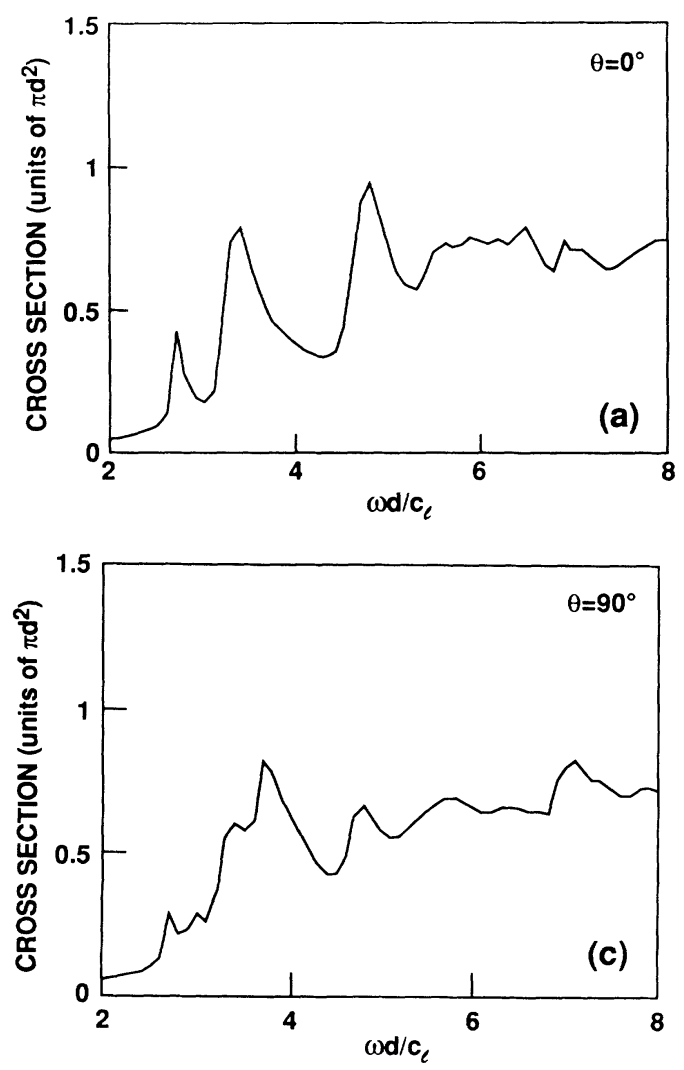

For the case of four-sphere scattering, the centers of the four spheres are placed at the corners of a tetrahedron. The $z$ axis is chosen to be perpendicular to one face of the tetrahedron and passes through one sphere center (Fig. 9). The incident wave is assumed to be in a plane perpendicular to one edge of the tetrahedron. The scattering cross section for different incident angles $\theta$ (between the direction of the incident wave and the $z$ axis), and the average over all incident angles, are given in Fig. 10. It can be seen that the splitting of the scattering peak is clearly evident. For all incident angles as well as the averaged result, a second scattering minimum appears which is at a frequency lower than that of the first minimum for the single-sphere scattering. This minimum results from the strong multiple-scattering coupling between neighboring spheres. In contrast to the two-sphere case, in the configuration of four spheres the coupling is strong for any incident angle because relative to each incident direction there are always pairs in the cluster which are oriented obliquely. From this calculation one concludes that the low-frequency mode propagates predominantly through spheres oriented obliquely relative to the propagation direction.

\section{DOS for a multiple-sphere scattering unit immersed in an effective medium}

In Fig. 11 (shown on p. 6520) we show the DOS for the case of a scattering unit with $N=2$ [see Fig. 2(b)]. The corresponding volume fraction is $\Phi=0.51$, and the max-
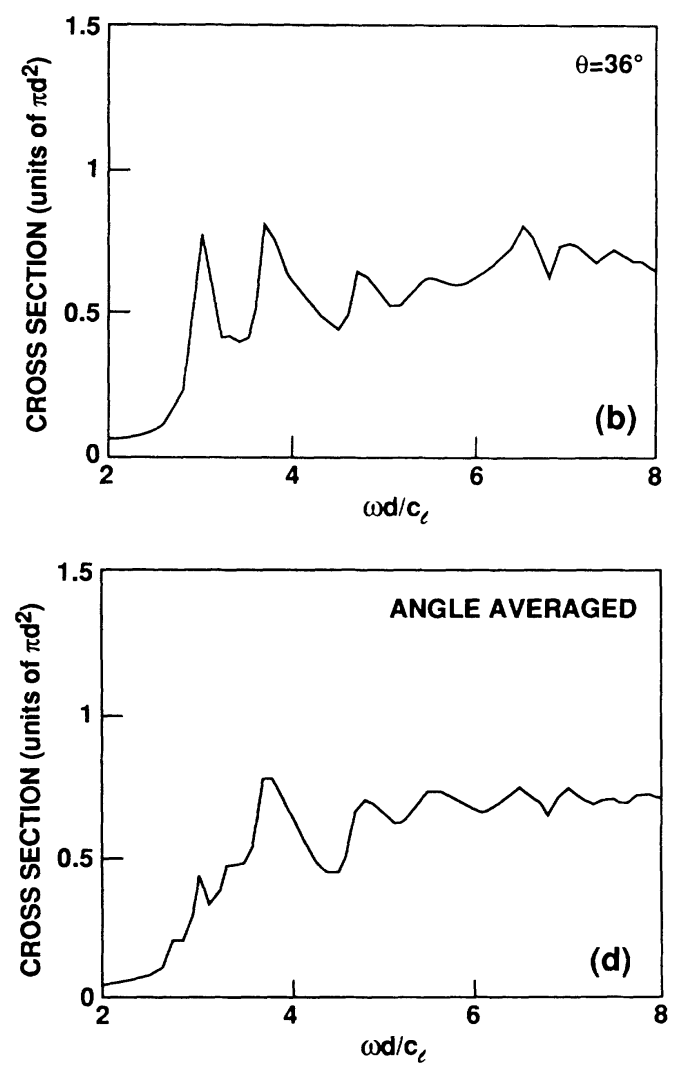

FIG. 10. Renormalized scattering cross section of four spheres immersed in liquid for three different incident angles: (a) $\theta=0^{\circ}$, (b) $\theta=36^{\circ}$, (c) $\theta=90^{\circ}$, and (d) the result of averaging over all angles. The angle $\theta$ is defined as in Fig. 9 . 
imum angular-momentum index $l_{0}$ used in the calculation is 12 . It can be seen that the result is very similar to that of the single-coated sphere in Fig. 3(b). However, there are several important differences. First, the width for both modes becomes larger than that for a single sphere, especially for the low-frequency mode around $q d=5$. This is in agreement with the experimental result that around $q d=5$ the low-frequency mode is very weak. Second, the peak position of the DOS for the lowfrequency mode is seen to move down in frequency around $q d=5$, thereby becoming closer to experimental data. This is due to the short-range-order effect that we have taken into account in our two-sphere calculation. Third, the higher-order gaps in the high-frequency mode become less distinct. This apparently also results from the interaction between the two spheres, and is closer to the experimental observation.

In order to delineate the short-range correlation effect even more clearly, we have carried out a calculation for a cluster of four spheres (Fig. 9) and ten spheres [see Fig. 2(c)]. In both case the scattering unit is assumed to be spherical in shape so as to avoid complex surface integrations. The configuration of four spheres, as described previously, is a tetrahedron. The configuration of the ten spheres is obtained from Monte Carlo simulated annealing by assuming a repulsive interaction among the spheres. Figures 12(b) and 12(c) show the results of foursphere and ten-sphere calculations, respectively, at a volume fraction $\Phi=0.38$. The maximum angular momentum $l_{0}$ used in the calculations is 10 and 7 for the case of four and ten spheres, respectively. Due to the limited angular-momentum components that one can include in the calculation, it is no longer possible to obtain accurate results for $q d>l_{0}$ as discussed below. We thus chose to compare calculated and experimental results at a fixed $q d<l_{0}$. At $q d=5.3$, the DOS is plotted as a function of reduced frequency $\omega d / c_{l}$. The same curve for a single-coated sphere is also shown in Fig. 12(a) for comparison. In Fig. 3(a) it is seen that at $q d=5.3$, there are four peaks of DOS. These are clearly evident in Fig. 12(a). Experimentally, however, the lower peaks are not identifiable and only two are evident. These are indicated by the two arrows. It can be seen that as $N$ increases from 4 to 10, the low-frequency mode becomes lower in frequency and both modes become broader than the single-coated-sphere case. These results thus not only confirm the short-range-order effect but also verify the physical picture presented by the single-coated-sphere case.

As stated in Sec. II, the nonvanishing scattering cross section at the antiresonance condition means that the modes are quasimodes. They have finite lifetimes, or finite frequency widths $\delta \omega$. Although our single-sphere calculations given excellent agreement in terms of dispersion relations, it cannot reproduce the widths of the two modes because of the lack of random scattering from neighboring spheres. The widths in the two-sphere calculation are better, but the peaks are still narrower compared with the experimental results. Our four-sphere calculation, however, does give results which are in reasonable agreement with the experiment. In Fig. 13 the quali- ty factor $\omega / \delta \omega$ is plotted as a function of reduced wave vector $q d$ for the two modes. The experimental results from Ref. [12] are also shown for comparison. The peak in the quality factor for the high-frequency mode around $q d \approx 2 \pi$ is reproduced. This could well be the result of constructive interference resulting from wave scattering by the basic scale $d$ of the medium.

The most serious source of error involved in our calculations is the angular-momentum cutoff imposed on the expansion of wave fields and in the displacement transformation of spherical waves [Eq. (3.24)]. This cutoff is necessitated by the computer memory limitation. For the case of a single sphere in fluid (Fig. 4), there is no displacement transformation involved in the calculation, and the error is checked to be negligible as long as the maximum $l_{0}$ exceeds $\omega d / c_{l}$. In order to check the error involved in the displacement transformation when the sum over $l$ is only up to $l_{0}$, we have repeated the single-

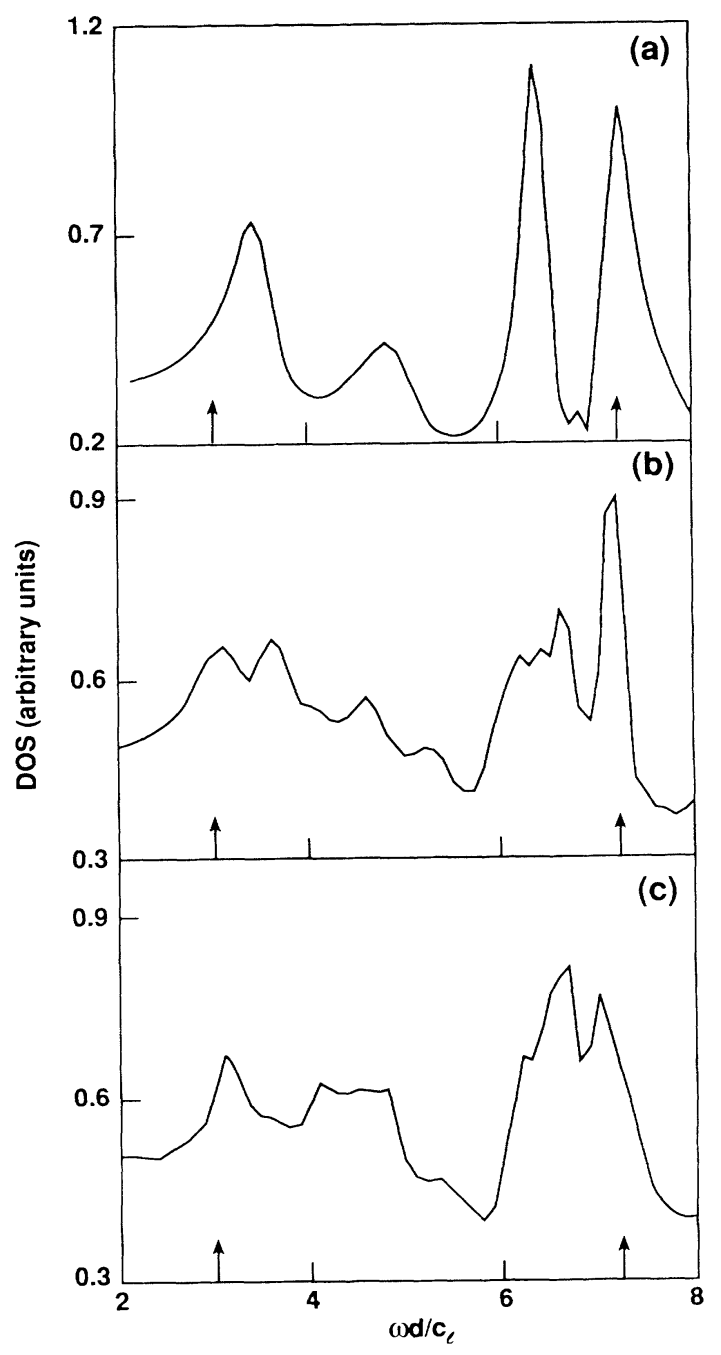

FIG. 12. The DOS vs reduced frequency $\omega d / c_{l}$ at a fixed reduced wave vector $q d=5.3$ and $\Phi=0.38$. (a) Single-coatedsphere result; (b) four-sphere result; (c) ten-sphere result. The arrows indicate the experimental peak positions. 


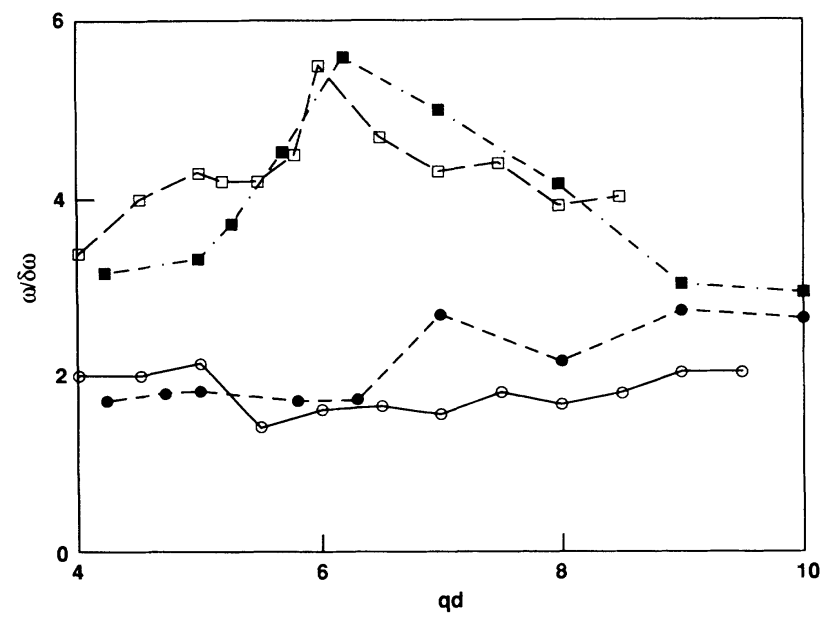

FIG. 13. The quality factor $\omega / \delta \omega$ for the two modes as a function of $q d$, calculated from the four-sphere scattering unit embedded in an effective medium with $\Phi=0.38$. Open squares and circles are the numerical results for the high-frequency and the low-frequency mode, respectively. Solid squares and circles correspond to experimental data.

sphere calculation by choosing the origin $O$ outside the sphere [see Fig. 14(a)], and use the transformation, Eq. (3.24), with the cutoff $l_{0}=7$. The total-scattering cross section is obtained as a function of frequency for two different incident angles $\theta=0^{\circ}$ and $60^{\circ}$ as well as for the average over al incident angles. The results are shown in Fig. 14(b). Theoretically, the scattering cross section should be independent of the incident angle, so the deviations from the accurate result [solid curve in Fig. 14(b), obtained from the calculation without the origin shift] for different incident angles give an indication of the error involved. It is seen that there is no apparent error up to $\omega d / c_{l}=l_{0}$, but for $\omega d / c_{l}>l_{0}$ the results for different incident angles clearly deviate from the accurate result. This simple criterion thus provides us with a simple rule of thumb for an a priori determination of $l_{0}$ to be used, as well as for judgment of accuracy in the final results.

\section{NUMERICAL SIMULATION IN TWO DIMENSIONS}

To further check our theory and the effect of fluid visciosity, numerical simulation of a two-dimensional system is carried out by using a finite-difference scheme on a staggered grid [21]. The model consists of a line (or point) time-harmonic source with frequency $\omega$ placed on one side (or center) of a $600 \times 600$ grid with about $20 \times 20$ solid disks in the system. The relevant first-order partial-differential equations are as follows. Inside the solid disks,

$$
\begin{aligned}
& \frac{\partial v_{i}}{\partial t}=\frac{1}{\rho} \frac{\partial \sigma_{i, k}}{\partial x_{k}}, \\
& \frac{\partial \sigma_{i, j}}{\partial t}=\kappa_{s} \delta_{i, j}+\mu_{s}\left(\frac{\partial v_{i}}{\partial x_{j}}+\frac{\partial v_{j}}{\partial x_{i}}-\frac{2}{3} \delta_{i, j} \frac{\partial v_{k}}{\partial x_{k}}\right) .
\end{aligned}
$$

For the liquid,

$$
\begin{aligned}
& \frac{\partial v_{i}}{\partial t}=\frac{1}{\rho_{l}} \frac{\partial \sigma_{i, k}}{\partial x_{k}} \\
& \sigma_{i, j}=-p \delta_{i, j}+\eta_{l}\left(\frac{\partial v_{i}}{\partial x_{j}}+\frac{\partial v_{j}}{\partial x_{i}}-\frac{2}{3} \delta_{i, j} \frac{\partial v_{k}}{\partial x_{k}}\right), \\
& \frac{\partial p}{\partial t}=-\kappa_{l} \frac{\partial v_{k}}{\partial x_{k}}
\end{aligned}
$$

Here $\kappa_{s}=\lambda_{s}+\frac{2}{3} \mu_{s}$ and $\kappa_{l}=\lambda_{l}$ are the bulk moduli of the solid and liquid, respectively, $\eta_{l}$ denotes the liquid visciosity, $p$ is the liquid pressure, $\sigma_{i, j}$ is the stress tensor, $v_{i}$ denotes velocity, indices $i, j, k$ denote the $x$ or $y$ component, and the summation is understood for repeated indices in a given term. The boundary conditions of velocity and traction continuity are applied across interfaces [22]. The discretized first-order partial-differential equa-

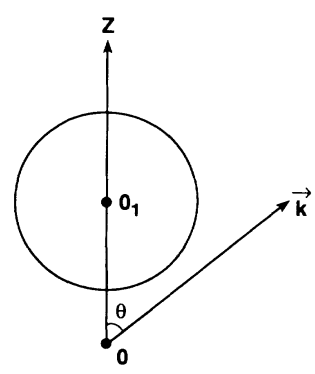

(a)

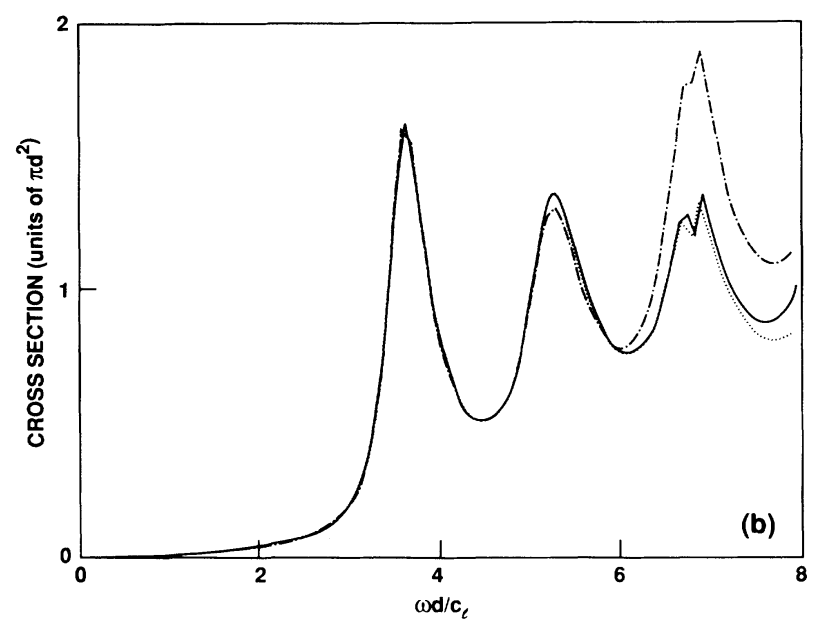

FIG. 14. (a) Sketch of the geometry used in the single-sphere scattering cross-section calculation by choosing the origin $O$ outside of the sphere. $\mathbf{k}$ is the incident wave vector. The distance $\overline{O O}_{1}$ is chosen to be $0.54 \mathrm{~d}$. (b) The results of the scattering cross section for different incident angles $\theta$. The dotteddashed curve is for $\theta=0^{\circ}$, the dotted curve is for $\theta=60^{\circ}$, and the solid curve denotes the accurate result. The scattering cross section should be $\theta$ independent. The deviations from the accurate result for $\omega d / c_{l}>7$ is a consequence of the finite angularmomentum cutoff, $l_{0}=7$, used in the displacement transformation calculation. 
tions are numerically integrated forward in time. After a suitable long integration time before wave front meets the boundary, the integration is stopped and the spatial distribution of $\boldsymbol{\nabla} \cdot \mathbf{v}$, which is proportional to the massdensity variation, is Fourier transformed into the $q$ domain. For a given frequency $\omega$, we can plot the Fourier amplitudes as a function of $q=|\mathbf{q}|$. Two peaks are clearly seen in the $q$ domain, thus directly verifying the existence of the two modes in a two-dimensional (2D) system. The dispersion relations of the two modes are shown in Fig. 15. Qualitative similarity to that shown in Fig. 3 is seen. The effect of decreasing the shear modulus is also examined, with exactly the same qualitative result as that shown in Fig. 6.

The effect of the fluid viscosity is examined. We found that unless the viscosity is unphysically large, its presence only causes minor corrections to the positions and widths of the two peaks. We can thus rule out the possibility of viscous coupling as a basic cause for the two observed modes.

Numerical simulation also enables us to obtain a microscopic picture of the two modes. By inverse Fourier transformation of each $q$ peak separately to the spatial domain, we get the mass-density variation $\nabla \cdot v$ in real space for each mode. Figure 16 gives a view of the $\boldsymbol{\nabla} \cdot \mathbf{v}$ amplitude for the two modes on a line perpendicular to the direction of wave propagation and passing through the centers of the two disks. It can be seen from the figure that there is a difference between the two modes at the interfaces between the solid disk and the liquid. For the high-frequency mode, the mass-density variation is seen to vary smoothly from one side to the other, whereas for the low-frequency mode there is a cusp at the interface, which is characteristic of the surface mode. The results of the numerical simulation thus support all the essential features of our physical picture.

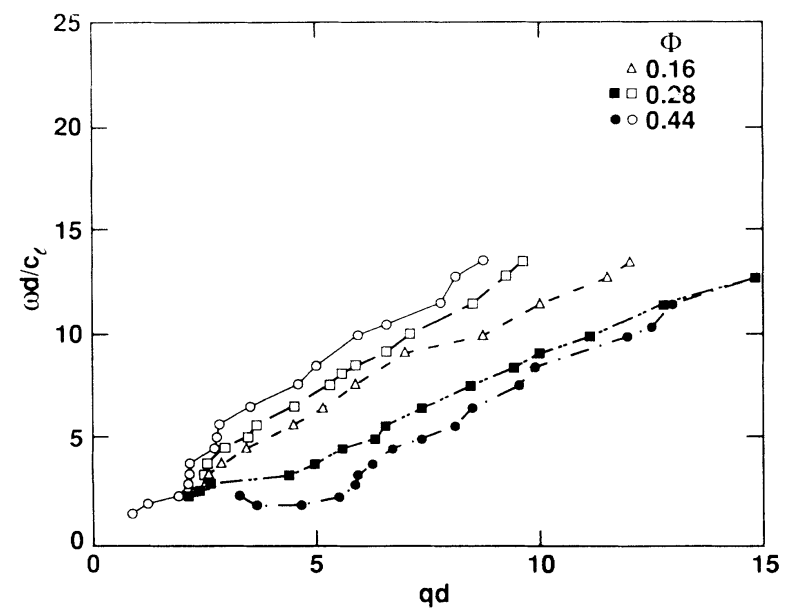

FIG. 15. The dispersion relations of the two quasimodes for a two-dimensional system of solid disks randomly dispersed in liquid. The results are obtained by numerical simulation as described in the text. The short-range-order effect for the lowfrequency mode is clearly seen at $\Phi=0.44$ as a dip in the dispersion relation.

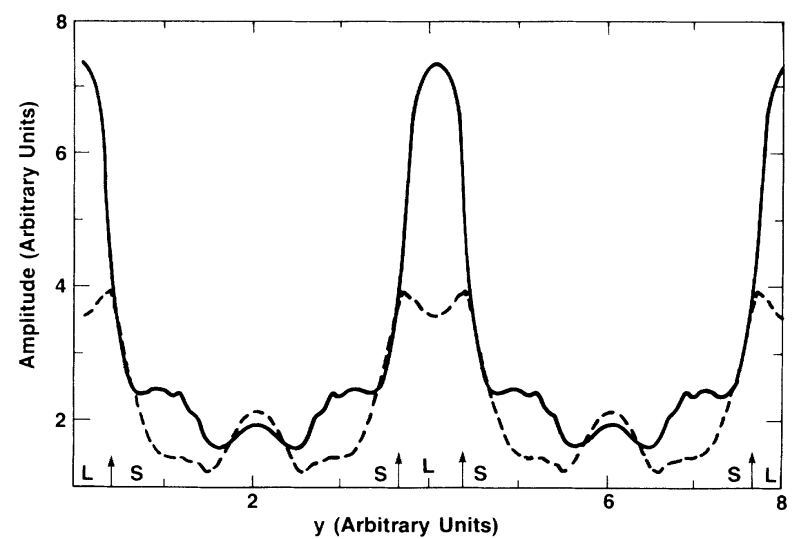

FIG. 16. Amplitude of $\boldsymbol{\nabla} \cdot \mathbf{v}$ for the two modes plotted along a line perpendicular to the direction of propagation and passing through the centers of two disks. The frequency used is $\omega d / c_{l} \simeq 7.5$. The dashed line denotes the low-frequency mode. The solid line denotes the high-frequency mode. Regions of solid and liquid are indicated by $S$ and $L$, respectively, at the bottom of the figure. The low-frequency mode is noted to have a cusp at the liquid-solid interface.

\section{THE ELECTROMAGNETIC CASE}

For a system of dielectric or metallic spheres randomly dispersed in a homogeneous dielectric medium, the effective dielectric constant $\epsilon_{m}$ in the long-wave length limit can be determined by the Maxwell-Garnett [23] equation. However, when the wavelength becomes comparable to the sphere size, resonance modes of the sphere will be excited, and quasimodes can appear as discussed before. In this section the emphasis will be on those aspects which are different from the acoustic-elastic case. The formulas for the scattering of a single sphere and a coated sphere can be found in Refs. [20] and [24]. Those related to the resonance modes may be found in Ref. [24].

\section{A. Scattering by a dielectric sphere}

In Fig. 17 the scattering cross section of a dielectric sphere (with dielectric constant $\epsilon_{s}$ or refractive index $n_{s}$ ) in vacuum (dielectric constant $\epsilon_{0}$ or refractive index $\left.n_{0}=1\right)$ is plotted as a function of reduced frequency $\omega d / c$ (where $c$ is the speed of light in vacuum and $d$ is the diameter of the sphere) for two different values of $\epsilon_{s}=1.77$ and 4.0. By comparing Fig. 17 with Fig. 4, two differences clearly emerge. First, the positions of the sharp scattering peaks in Fig. 17 are seen to be irregular, and the difference between the peak and valley is not as large as that of Fig. 4. Second, large dips (black arrows in Fig. 17) are seen to appear periodically in the electromagnetic case.

The first difference originates from the different physical origins of the resonance modes in the two cases. The resonance modes of an elastic sphere in fluid are Stoneley surface modes. Here they are bulk resonance modes, and the coupling to the incident wave could be smaller if the modes are bulk in nature. The second feature, that of the large dips, is seen to occur approximately at those fre- 
quencies which satisfy the relation

$$
\frac{\omega}{c}\left(n_{s}-n_{0}\right) d=2 m \pi
$$

where $m$ is an integer. Physically, when the phase difference between the wave passing through the sphere and the wave passing through the same distance $d$ in the matrix is $2 m \pi$, the scattering is minimum due to the constructive interference in the forward direction. The same kind of large dip feature is seen for the scattering of a liquid sphere immersed in a different fluid. This could be due to the fact that in this case, the resonances are again bulk modes as opposed to surface modes.

\section{B. Scattering by a metallic sphere}

In Fig. 18 we give the results of scattering by a metallike and a metallic sphere in vacuum. Figure 18(a) corresponds to the case where the dielectric constant of the sphere is negative but independent of frequency, $\epsilon_{s}=-3.24$. The result is seen to be very different from
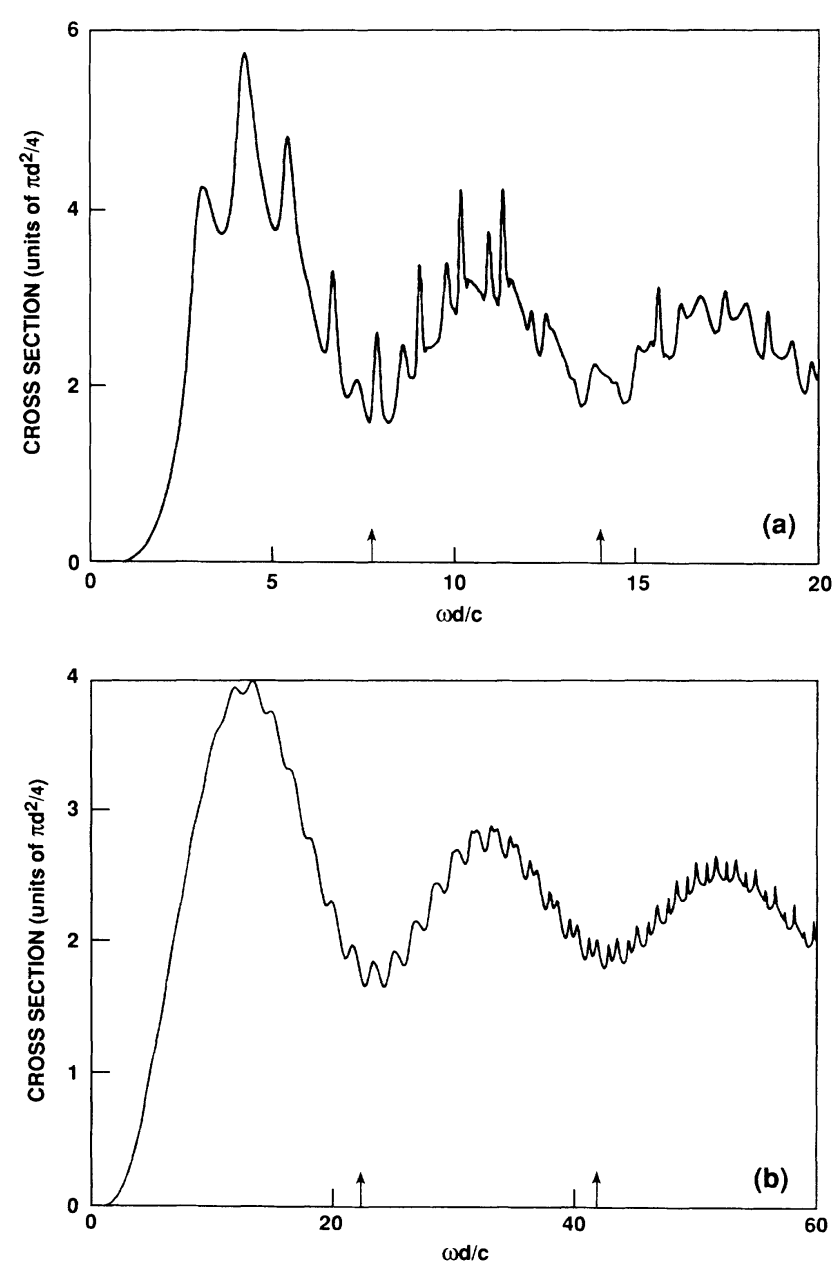

FIG. 17. Renormalized cross section of electromagneticwave scattering from a dielectric sphere, plotted as a function of $\omega d / c$. Here $c$ denotes the speed of light in vacuum. (a) The sphere dielectric constant is $\epsilon_{s}=4.0$. (b) $\epsilon_{s}=1.77$. The background dielectric constant is $\epsilon_{0}=1.0$ in both cases. The arrows indicate the positions of the dips. the case of $\epsilon_{s}>0$ as shown in Fig. 17. The scattering peaks are equally spaced, similar to the case of acousticwave scattering by an elastic sphere. This may be explained by the fact that the condition for fitting a standing wave on the sphere surface is given by

$$
\pi d=2 \pi m c_{\text {surf }} / \omega,
$$

where $m$ is an integer. From Eq. (6.2) we get the frequency separation in reduced units as $2 c_{\text {surf }} / c=1.66$. This is in excellent agreement with what is seen in Fig. 18(a). Here the surface mode speed is given by

$$
c_{\text {surf }}=c\left(\frac{\epsilon_{s}+\epsilon_{0}}{\epsilon_{s} \epsilon_{0}}\right)^{1 / 2},
$$

where the condition for the existence of a surface mode is

$$
\epsilon_{s} \epsilon_{0}<0 \text {, and } \epsilon_{s}+\epsilon_{0}<0 \text {. }
$$

For the parameters used in the calculation of Fig. 18(a), we have $c_{\text {surf }}=0.83 c$.

The dielectric constant of the Drude model is given by

$$
\epsilon_{s}=1-\omega_{p}^{2} / \omega^{2}
$$

where $\omega_{p}$ is the plasmon frequency. Now the scattering cross section is no longer dependent on only one parameter $\omega d / c$ as in the case of a constant $\epsilon_{s}$. There are two parameters, $\omega d / c$ and $\omega_{p}$, or $\omega / \omega_{p}$ and $\omega_{p} d / c$. In Figs. $18(\mathrm{~b}), 18(\mathrm{c})$, and $18(\mathrm{~d})$, the scattering cross sections are plotted as a function of $\omega / \omega_{p}$ for three different reduced diameters of the sphere $\omega_{p} d / c=0.4,2.0$, and 12.0, respectively. For small metallic spheres, $\omega_{p} d / c$ is much smaller than 1, the sharp resonance peak in Fig. 18(b) corresponds to the surface mode of a small particle [19]. The resonance peak occurs at $\omega / \omega_{p}=1 / \sqrt{3}$. As the sphere size increases, more resonance modes appear. These modes may be viewed similarly as standing waves formed at the surface. Because $\epsilon_{s}$ now depends on frequency, however, these modes are no longer equally spaced. By using the condition of Eq. (6.4) and Eq. (6.5), it is easily seen that these surface modes exist only in the region $\omega / \omega_{p}<1 / \sqrt{2}$ for $\epsilon_{0}=1$. As frequency increases beyond this critical value the surface mode disappears. Since $\epsilon_{s}$ approaches 1 as frequency increases, the scattering is seen to become weaker at high frequencies.

\section{DOS for a single-coated dielectric sphere embedded in an effective medium}

In the same way as in Sec. III, consider the model in which a coating with the same dielectric constant as the matrix material envelopes the sphere, and the coated sphere is embedded in a homogeneous medium of dielectric constant $\epsilon_{m}$. The wave vector in the effective medium is related to dielectric constant by the relation $k_{m}=\sqrt{\epsilon_{m}} \omega / c$. Under the long-wavelength limit (wavelength much greater $d$ ), the value of $k_{m}$ or $\epsilon_{m}$ is determined by the condition of vanishing forward-scattering amplitude. This results in the equation 


$$
\left(\frac{b}{a}\right)^{3} \frac{\epsilon_{m}-\epsilon_{0}}{\epsilon_{0}-\epsilon_{s}}+\frac{\epsilon_{m}+2 \epsilon_{0}}{\epsilon_{s}+2 \epsilon_{0}}=0,
$$

where $a$ and $b$ are the radii of the sphere and the sphere plus coating, respectively. Since $(a / b)^{3}$ gives the volume fraction of the dielectric spheres, Eq. (6.6) may be seen as equivalent to the Maxwell-Garnett theory [23], where

$$
\epsilon_{m}=\epsilon_{0} \frac{(1+2 \Phi) \epsilon_{s}+2(1-\Phi) \epsilon_{0}}{(1-\Phi) \epsilon_{s}+(2+\Phi) \epsilon_{0}}
$$

For the case of dielectric inclusions, $\epsilon_{s}>0$, one expects a well-defined mode at low frequencies whose dispersion relation is given by $\omega=c k_{m} / \sqrt{\epsilon_{m}}$. As frequency increases, the scattering from spheres becomes strong, and this mode should weaken and gradually disappear. This is clearly seen in Fig. 19, where we give the results of the DOS calculation for $\epsilon_{s}=4$. In this case there is no corresponding low-frequency mode as in the colloidal systems.

Except for the mode described above, our calculation shows the existence of a quasimode which appears when the frequency exceeds some critical value and satisfies the condition arising from Eq. (6.1). In the presence of other
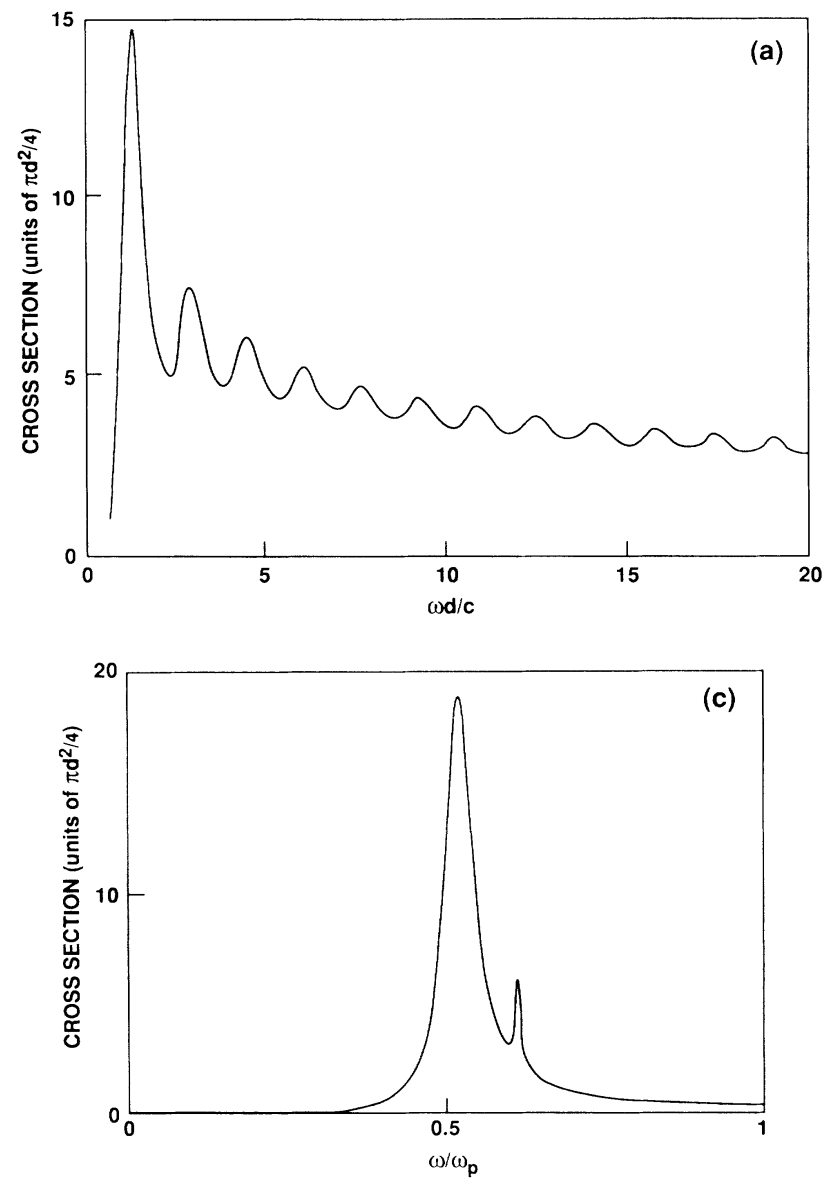

spheres, this condition becomes

$$
2 n_{s} a \omega / c+2 n_{0}(b-a) \omega / c-2 k_{m} b=2 m \pi,
$$

where $k_{m}$ represents the effective-medium property resulting from multiple scattering by all the spheres. According to Eq. (6.8), the first minimum in scattering cross section occurs at $m=1, k_{m}=0$, and

$$
\omega=\pi c /\left[a n_{s}+n_{0}(b-a)\right] .
$$

The frequency which satisfies the dip condition is a function of $k_{m}$. Therefore, for each value of $m$ there is a band, with each band forming a mode whose group velocity is given by

$$
\frac{d \omega}{d k_{m}}=c b /\left[a n_{s}+(b-a) n_{0}\right] \text {. }
$$

These results are clearly seen in Fig. 19.

One way in which our results can be compared with experiment is through the effective dielectric constant defined by $\bar{\epsilon}=\left(k_{\max } / \omega_{\max }\right)^{2} c^{2}$, where $k_{\max }$ and $\omega_{\max }$ denote the peak position of the DOS. A necessary condition for the unique definition of $\bar{\epsilon}(\omega)$ is that for every $\omega_{\max }$ there should be only one $k_{\max }$, i.e., a maximum of
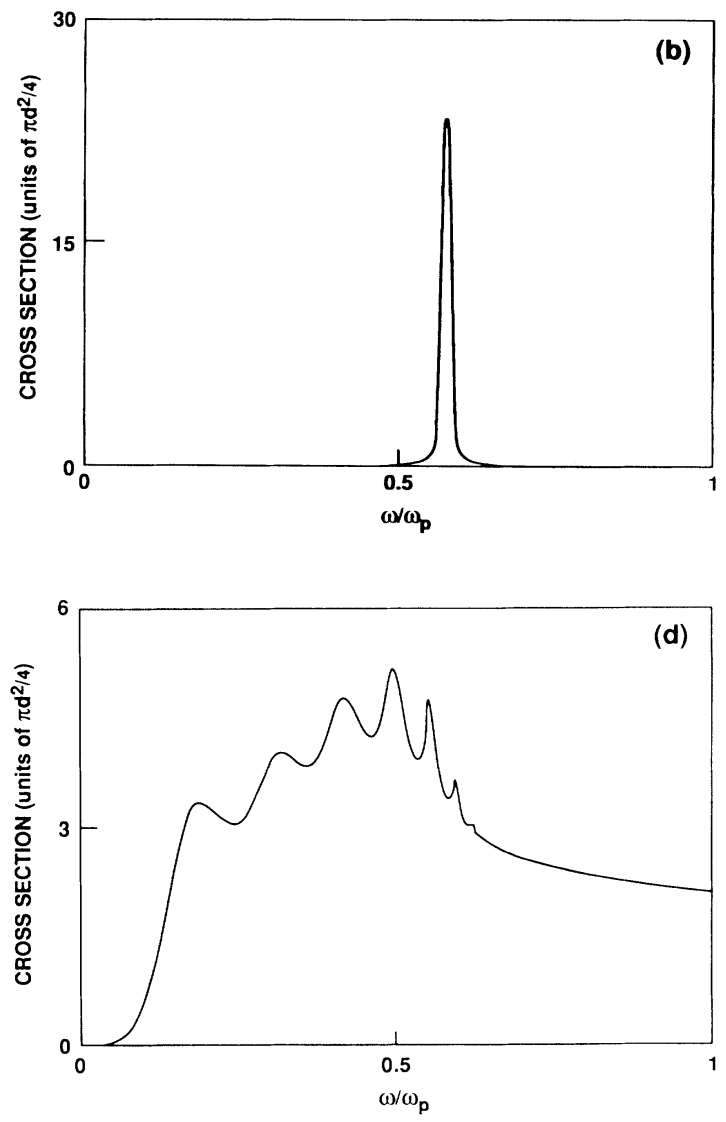

FIG. 18. Renormalized scattering cross section of an electromagnetic wave for a metal-like sphere. (a) corresponds to the case of the frequency-independent negative dielectric constant $\epsilon_{s}=-3.24$. (b)-(d) correspond to the results where the sphere has the Drude model dielectric constant $\epsilon_{s}=1-\omega_{p}^{2} / \omega^{2}$ for reduced sphere diameters $\omega_{p} d / c=0.4,2.0$, and 12.0, respectively. Here $\omega_{p}$ is the plasmon frequency of the metallic sphere, $c$ denotes the speed of light in vacuum. The background dielectric constant is $\epsilon_{0}=1.0$. 


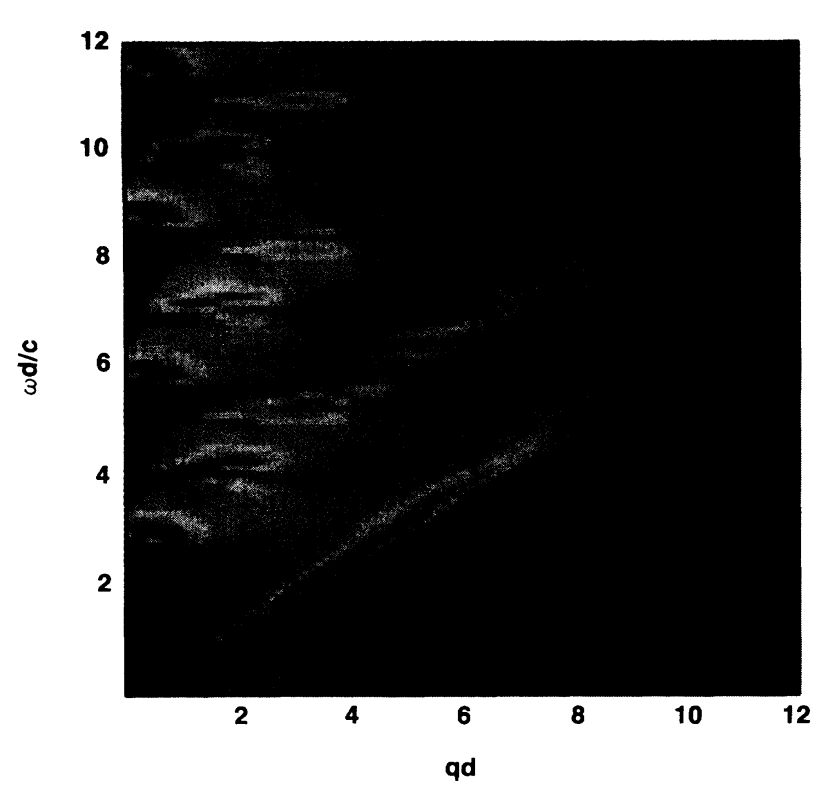

FIG. 19. The DOS of a single-coated dielectric sphere in effective medium, plotted as a function of reduced wave vector $q d$ and reduced frequency $\omega d / c$. The color scheme is the same as that of Fig. 3. The dielectric constants of the sphere and the matrix are $\epsilon_{s}=4.0$ and $\epsilon_{0}=1.0$, respectively, and $\Phi=0.3$.

one mode for each frequency. From Fig. 19 it is clear that the condition is indeed satisfied for $\omega d / c<\pi$, where our $\bar{\epsilon}(\omega)$ corresponds to nothing more than the MaxwellGarnett theory, the validity of which has been extensively examined in the literature. For $\omega d / c>\pi$, however, the uniqueness condition breaks down, and one can no longer define an effective dielectric constant. Nevertheless, it is precisely in this regime that we expect the quasimodes to occur. To our knowledge, experimental data on the existence of electromagnetic quasimodes in random dispersed media is still lacking. Our results may thus provide the guidance for their location.

\section{DOS for a single-coated metallic sphere embedded in an effective medium}

If the inclusion is metallic, $\epsilon_{s}$ may be less than zero, and the average dielectric constant $\epsilon_{m}$ give by Eq. (6.7) can be negative, implying no propagating mode in the system. For a given volume fraction and the matrix dielectric constant $\epsilon_{0}(>0)$, there exists two critical values of $\epsilon_{s}, \epsilon_{s}^{u}$ and $\epsilon_{s}^{d}$, at which $\epsilon_{m}$ changes sign:

$$
\begin{aligned}
& \epsilon_{s}^{u}=-\epsilon_{0} \frac{2(1-\Phi)}{1+2 \Phi}, \\
& \epsilon_{s}^{l}=-\epsilon_{0} \frac{2+\Phi}{1-\Phi}
\end{aligned}
$$

For $\epsilon_{s}^{l}<\epsilon_{s}<\epsilon_{s}^{u}, \epsilon_{m}<0$. Otherwise, $\epsilon_{m}$ is positive. For the Drude model given by Eq. (6.5), there exist correspondingly two frequencies $\omega^{u}$ and $\omega^{l}$ by

$$
\omega^{u}=\frac{\omega_{p}}{\sqrt{\left[1+2 \epsilon_{0}(1-\Phi) /(1+2 \Phi)\right]}},
$$

$$
\omega^{l}=\frac{\omega_{p}}{\sqrt{\left.1+\epsilon_{0}(2+\Phi) /(1-\Phi)\right]}},
$$

where in the region $\omega^{l}<\omega<\omega^{u}$, the effective $\epsilon_{m}<0$. As $\Phi$ approaches zero, both $\omega^{u}$ and $\omega^{l}$ approach $\omega_{p} / \sqrt{1+2 \epsilon_{0}}$, which is just the Mie resonance frequency. For finite volume fraction of metal spheres, there is a gap in the excitation spectrum of the system. Here $\omega^{u}$ represents the plasmon frequency of the metallic colloidal system, and $\omega^{l}$ is seen to correspond to the frequency of the dielectric anomaly, a well-known effect in the optics of granular metals [25]. In Fig. 20(a), the DOS is plotted versus the reduced wave vector $k_{m} c / \omega_{p}$ and reduced fre-
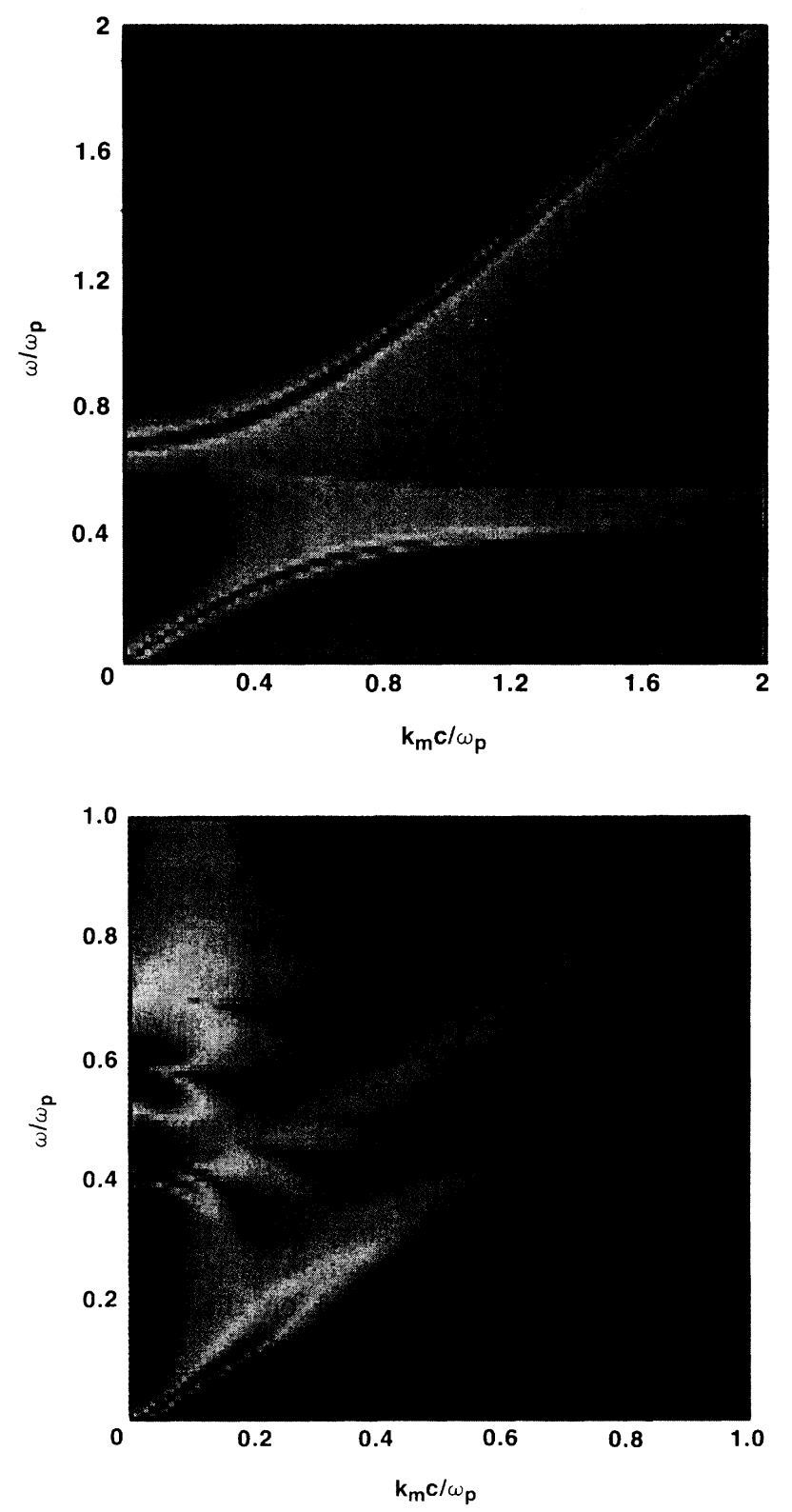

FIG. 20. The DOS for a single-coated metallic sphere in effective medium with $\Phi=0.3$. The color scheme is the same as that of Fig. 3. (a) The reduced sphere size is $\omega_{p} d / c=2.0$. (b) The reduced sphere size is $\omega_{p} d / c=12.0$. 
quency $\omega / \omega_{p}$ for a sphere size $\omega_{p} d / c=2.0$. The volume fraction is 0.30 . The two red curves correspond to the modes of $\omega<\omega^{l}$ and $\omega>\omega^{u}$ in the long-wavelength limit. In fact, at this sphere size, one additional resonant mode already exists as shown in Fig. 18(c), and the yellow band in the region of the gap, shown in Fig. 20(a), indicates that the same resonances on neighboring spheres can couple, giving rise to a weak quasimode. If one defines the effective dielectric constant $\bar{\epsilon}$ as discussed before, then the existence of the mode in the gap already indicates departure from the Maxwell-Garnett theory. As the sphere size increases, more modes are excited in the gap region. The coupling between the spheres also tends to increase with increasing sphere size because of the stronger surface current. The results shown in Fig. 20(b) is the case for $\omega_{p} d / c=12.0$, with volume fraction $\Phi=0.3$. It is clear that in this case the modes cover the original gap region of Fig. 20(a). Another significant difference between the electromagnetic case discussed in this section and the acoustic wave in the colloidal system, lies in the existence of different polarizations for the electromagnetic wave, which may give rise to depolarization scattering. As a result, the strength of the quasimodes predicted in this section, based only on one coated-sphere calculation, could be appreciably decreased. What is clear from Fig. 20(b) is that for this sphere size, an effective $\bar{\epsilon}$ is meaningful only at $\omega / \omega_{p}$ approximately less than 0.2. Again, just as in the dielectric sphere case, the prediction of quasimodes awaits further experimental verification.

\section{CONLCUDING REMARKS}

In this work we have presented a simple GCPA approach, based on the principle of locating the minima of $\langle T\rangle$, for identifying the quasiwave modes in random dispersed media and the calculation of their dispersion relations. The validity of this approach is verified by comparison with experimental results as well as by multiple-scattering calculations and numerical simulation. The results obtained show that in the frequency regime where the wavelength is comparable to or smaller than the scale of the inhomogeneities, there can be significant new phenomena arising from the coherent coupling of resonances on neighboring particles.

It is important to point out the limitation of our approach, which is inherently related to the fact that the GCPA studies only the properties of the averaged single Green's function. As a result, the diffusive-transport characteristics and the localization properties of these quasiwave modes, which depend on the averaged two
Green's functions, are beyond the present formalism and remain a topic for further investigation.

\section{ACKNOWLEDGMENTS}

The authors wish to acknowledge many helpful discussions with D. Weitz and Z. Q. Zhang.

\section{APPENDIX A: GREEN'S FUNCTION AND THE SCATTERING OPERATOR FOR A SINGLE-COATED SPHERE}

In this appendix we summarize the calculation of the Green's function and the scattering operator for a solid sphere with a liquid coating embedded in an effectivemedium fluid background. Schwartz and Plona [3] have obtained the result for the case of a solid sphere without coating. We extend their calculation to the case of a coated sphere.

As described in Sec. III, the GF $G_{\psi}^{(s)}\left(\mathbf{r}_{1}, \mathbf{r}_{2}\right)$ represents the compressional response at $\mathbf{r}_{1}$, given a compressional source at $\mathbf{r}_{2}$. The shear part of the GF is given by $G_{\xi}^{(s)}\left(\mathbf{r}_{1}, \mathbf{r}_{2}\right) . G_{\psi}^{(s)}\left(\mathbf{r}_{1}, \mathbf{r}_{2}\right)$ and $G_{\xi}^{(s)}\left(\mathbf{r}_{1}, \mathbf{r}_{2}\right)$ satisfy Eqs. (3.14) and (3.15), respectively. They are coupled by the boundary conditions at the surfaces $r_{1}=a$ and $r_{1}=b$. The boundary conditions are as follows. For the solid-fluid interface, normal displacements and normal stresses are continuous, with vanishing tangential stresses. For the fluid-fluid interface, normal displacements and normal stresses are continuous. By using the expansion (3.16), it is straightforward to show that $g_{\psi l}^{(s)}\left(r_{1}, r_{2}\right)$ satisfies the equation

$$
\begin{array}{r}
{\left[\frac{\partial^{2}}{\partial r_{1}^{2}}+\frac{2}{r_{1}} \frac{\partial}{\partial r_{1}}+k_{\left(r_{1}\right)}^{2}-l(l+1) / r_{1}^{2}\right] g_{\psi l}^{(s)}\left(r_{1}, r_{2}\right)} \\
=\delta\left(r_{1}-r_{2}\right) / r_{1}^{2},
\end{array}
$$

where $k\left(r_{1}\right)$ denotes the corresponding longitudinal wave vector in the three regions of $r_{1}<a, a<r_{1}<b$, and $r_{1}>b$. For $g_{\xi l}^{(s)}\left(r_{1}, r_{2}\right)$ the relevant equation is

$$
\begin{gathered}
{\left[\frac{\partial^{2}}{\partial r_{1}^{2}}+\frac{2}{r_{1}} \frac{\partial}{\partial r_{1}}+k_{t}^{2}-l(l+1) / r_{1}^{2}\right] g_{\xi l}^{(s)}\left(r_{1}, r_{2}\right)=0,} \\
r_{1} \leq a, \\
g_{\xi l}^{(s)}\left(r_{1}, r_{2}\right)=0, \quad r_{1}>a .
\end{gathered}
$$

Here $k_{t}$ is the transverse-wave vector inside the solid sphere. The solutions of Eqs. (A1) and (A2) are spherical Bessel functions. The solutions of Eq. (A1) are listed below for the three cases. For $r_{2}>b$,

$$
g_{\psi l}^{(s)}\left(r_{1}, r_{2}\right)=\left\{\begin{array}{l}
-i k_{m} a_{l} j_{l}\left(k_{p} r_{1}\right) h_{l}\left(k_{m} r_{2}\right), \quad r_{1} \leq a, \\
-i k_{m} f_{l}\left[j_{l}\left(k_{l} r_{1}\right)+A_{l} h_{l}\left(k_{l} r_{1}\right)\right] h_{l}\left(k_{m} r_{2}\right), \quad a<r_{1} \leq b, \\
-i k_{m}\left[j_{l}\left(k_{m} r_{<}\right)+\bar{A}_{l} h_{l}\left(k_{m} r_{<}\right)\right] h_{l}\left(k_{m} r_{>}\right), \quad r_{1}>b .
\end{array}\right.
$$

For $a<r_{2} \leq b$, 


$$
g_{\psi l}^{(s)}\left(r_{1}, r_{2}\right)=\left\{\begin{array}{l}
-i k_{l} \Delta_{l} j_{l}\left(k_{p} r_{1}\right)\left[\Gamma_{l} j_{l}\left(k_{l} r_{2}\right)+h_{l}\left(k_{l} r_{2}\right)\right], \quad \gamma_{1} \leq a \\
-i k_{l} \Sigma_{l}\left[j_{l}\left(k_{l} r_{<}\right)+A_{l} h_{l}\left(k_{l} r_{<}\right)\right]\left[\Gamma_{l} j_{l}\left(k_{l} r_{>}\right)+h_{l}\left(k_{l} r_{>}\right)\right], \quad a<r_{1} \leq b \\
-i k_{l} \delta_{l} h_{l}\left(k_{m} r_{1}\right)\left[j_{l}\left(k_{l} r_{2}\right)+A_{l} h_{l}\left(k_{l} r_{2}\right)\right], \quad r_{1}>b .
\end{array}\right.
$$

For $r_{2} \leq a$,

$$
g_{\psi l}^{(s)}\left(r_{1}, r_{2}\right)= \begin{cases}-i k_{p} j_{l}\left(k_{p} r_{<}\right)\left[h_{l}\left(k_{p} r_{>}\right)+B_{l} j_{l}\left(k_{p} r_{>}\right)\right], & r_{1} \leq a \\ -i k_{p} E_{l}\left[\Gamma_{l} j_{l}\left(k_{l} r_{1}\right)+h_{l}\left(k_{l} r_{1}\right)\right] j_{l}\left(k_{p} r_{2}\right), & a<r_{1} \leq b \\ -i k_{p} \bar{E}_{l} h_{l}\left(k_{m} r_{1}\right) j_{l}\left(k_{p} r_{2}\right), \quad r_{1}>b & \end{cases}
$$

In the above equations, $r_{<}$and $r_{>}$represent the smaller and larger one of $r_{1}$ and $r_{2}$, respectively. All the coefficients are determined by fitting the boundary conditions at the two surfaces $r_{1}=a$ and $r_{1}=b$, and they are given in the following. For simplicity, we discard all the subscripts $l$ of angular-momentum label. The subscripts $p, t, f$, and $m$ are used to represent the arguments of the corresponding spherical Bessel functions, and we define $x_{p}=k_{p} r, x_{t}=k_{t} r, x_{f}=k_{l} r$, $x_{m}=k_{m} r$

$$
\begin{aligned}
& A_{l}=-\frac{U^{(1)} \beta_{2}+l(l+1) \alpha_{1} V^{(1)}}{U^{(3)} \beta_{2}+l(l+1) \alpha_{1} V^{(3)}}, \\
& \alpha_{1}=\left.2\left(j_{p}^{\prime} / x_{p}-j_{p} / x_{p}^{2}\right)\right|_{r=a}, \\
& \beta_{2}=[l(l+1)-2] j_{t} / x_{t}^{2}+\left.j_{t}^{\prime \prime}\right|_{r=a}, \\
& U^{(1)}=Q_{0}\left[-j_{p}^{\prime \prime}+Q_{1} j_{p}\right] j_{f}^{\prime} / x_{f}-j_{p}^{\prime} j_{f} /\left.x_{p}\right|_{r=a}, \\
& V^{(1)}=Q_{0}\left(j_{t} / x_{t}\right)^{\prime} j_{f}^{\prime} / x_{f}+j_{f} j_{t} /\left.x_{t}^{2}\right|_{r=a}, \\
& Q_{0}=2 \rho c_{t}^{2} / \rho_{l} c_{l}^{2}, \quad Q_{1}=\left(c_{p}^{2}-2 c_{t}^{2}\right) / 2 c_{t}^{2} .
\end{aligned}
$$

$U^{(3)}$ and $V^{(3)}$ have the same form as $U^{(1)}$ and $V^{(1)}$, respectively, except $j_{f}$ is replaced by $h_{f}$ and $j_{f}^{\prime}$ is replaced by $h_{f}^{\prime}$ :

$$
\begin{aligned}
& \bar{A}_{l}=\frac{\left(k_{m} \rho_{l} j_{m}^{\prime} j_{f}-k_{l} \rho_{m} j_{m} j_{f}^{\prime}\right)+\left(k_{m} \rho_{l} j_{m}^{\prime} h_{f}-k_{l} \rho_{m} j_{m} h_{f}^{\prime}\right) A_{l}}{\left(k_{m} \rho_{l} h_{m}^{\prime} j_{f}-k_{l} \rho_{m} h_{m} j_{f}^{\prime}\right)+\left.\left(k_{m} \rho_{l} h_{m}^{\prime} h_{f}-k_{l} \rho_{m} h_{m} h_{f}^{\prime}\right) A_{l}\right|_{r=b},} \\
& f_{l}=\frac{\rho_{m}}{\rho_{l}}\left(j_{m}+\bar{A}_{l} h_{m}\right) /\left.\left(j_{f}+A_{l} h_{f}\right)\right|_{r=b}, \\
& a_{l}=\left.\frac{x_{f} f_{l}\left(j_{f}^{\prime}+A_{l} h_{f}^{\prime}\right)}{\left[j_{p}^{\prime} / x_{p}-l(l+1) \alpha_{1} j_{t} /\left(\beta_{2} x_{t}^{2}\right)\right] x_{p}^{2}}\right|_{r=a}, \\
& \Gamma_{l}=-\left(k_{m} \rho_{l} h_{f} h_{m}^{\prime}-k_{l} \rho_{m} h_{f}^{\prime} h_{m}\right) /\left.\left(k_{m} \rho_{l} j_{f} h_{m}^{\prime}-k_{l} \rho_{m} j_{f}^{\prime} h_{m}\right)\right|_{r=b}, \\
& \Sigma_{l}=1 /\left(1-A_{l} \Gamma_{l}\right), \\
& \delta_{l}=\frac{\rho_{l}}{\rho_{m}}\left(\Gamma_{l} j_{f}+h_{f}\right) \Sigma_{l} /\left.h_{m}\right|_{r=b}, \\
& \Delta_{l}=x_{f}\left(j_{f}^{\prime}+A_{l} h_{f}^{\prime}\right) \Sigma_{l} /\left.\left\{\left[j_{p}^{\prime} / x_{p}-l(l+1) \alpha_{1} j_{t} /\left(\beta_{2} x_{t}^{2}\right)\right] x_{p}^{2}\right\}\right|_{r=a}, \\
& B_{l}=-\frac{\beta_{2} W_{c}^{(3)}+l(l+1) \alpha_{1}^{(3)} V_{c}^{(3)}}{\beta_{2} U_{c}^{(3)}+l(l+1) \alpha_{1} V_{c}^{(3)}}, \\
& W_{c}^{(3)}=Q_{0}\left(-h_{p}^{\prime \prime}+Q_{1} h_{p}\right) h_{f}^{\prime} / x_{f}-f_{c l} h_{p}^{\prime} h_{f} /\left.x_{p}\right|_{r=a}, \\
& V_{c}^{(3)}=Q_{0}\left(j_{t} / x_{t}\right)^{\prime} h_{f}^{\prime} / x_{f}+f_{c l} j_{t} h_{f} /\left.x_{t}^{2}\right|_{r=a}, \\
& U_{c}^{(3)}=Q_{0}\left(-j_{p}^{\prime \prime}+Q_{1} j_{p}\right) h_{f}^{\prime} / x_{f}-f_{c l} j_{p}^{\prime} h_{f} /\left.x_{p}\right|_{r=a}, \\
& f_{c l}=\left(1+\Gamma_{l} j_{f} / h_{f}\right) /\left.\left(1+\Gamma_{l} j_{f}^{\prime} / h_{f}^{\prime}\right)\right|_{r=a}, \\
& \alpha_{1}^{(3)}=\left.2\left(h_{p}^{\prime} / x_{p}-h_{p} / x_{p}^{2}\right)\right|_{r=a}, \\
& E_{l}=\left.\frac{\left(h_{p}^{\prime}+B_{l} j_{p}^{\prime}\right) x_{p}-l(l+1)\left(\alpha_{1}^{(3)}+B_{l} \alpha_{1}\right) j_{t} x_{p}^{2} /\left(\beta_{2} x_{t}^{2}\right)}{\left(h_{f}^{\prime}\right) x_{f}}\right|_{r},
\end{aligned}
$$




$$
\bar{E}_{l}=\frac{\rho_{f}}{\rho_{m}}\left(\Gamma_{l} j_{f}+h_{f}\right) E_{l} /\left.h_{m}\right|_{r=b}
$$

The Green's function can be expanded in the momentum space as follows:

$$
G_{\psi}\left(\mathbf{p}_{1}, \mathbf{p}_{2}\right)=(4 \pi)^{2} \sum_{l, m} g_{\psi l}\left(p_{1}, p_{2}\right) Y_{l, m}\left(\hat{\mathbf{p}}_{1}\right) Y_{l, m}^{*}\left(\hat{\mathbf{p}}_{2}\right),
$$

where $g_{\psi l}\left(p_{1}, p_{2}\right)$ is given by

$$
g_{\psi l}\left(p_{1}, p_{2}\right)=\int_{0}^{\infty} \int_{0}^{\infty} r_{1}^{2} j_{l}\left(p_{1} r_{1}\right) r_{2}^{2} j_{l}\left(p_{2} r_{2}\right) g_{\psi l}\left(r_{1}, r_{2}\right) d r_{1} d r_{2} .
$$

The scattering matrix defined in Eq. (3.2) can be similarly expanded in angular-momentum space as

$$
T^{(s)}\left(\hat{\mathbf{p}}_{1}, \mathbf{p}_{2}\right)=(4 \pi)^{2} \sum_{l, m} T_{l}^{(s)}\left(p_{1}, p_{2}\right) Y_{l, m}\left(\hat{\mathbf{p}}_{1}\right) Y_{l, m}^{*}\left(\hat{\mathbf{p}}_{2}\right)
$$

Finally the scattering matrix $T_{l}^{(s)}\left(p_{1}, p_{2}\right)$ is given by

$$
\begin{aligned}
T_{l}^{(s)}\left(p_{1}, p_{2}\right)= & \left\{J_{p_{1}, p_{2}}^{b} /\left(p_{1}^{2}-k_{m}^{2}\right)-J_{p_{1}, p_{2}}^{a} /\left(p_{1}^{2}-k_{p^{2}}^{2}\right)-\bar{J}_{p_{1}, p_{2}} /\left(p_{1}^{2}-k_{l}^{2}\right)\right. \\
& -i k_{p} J_{p_{2}, k_{p}}^{a}\left[H_{p_{1}, k_{p}}^{a}+B_{l} J_{p_{1}, k_{p}}^{a}+\left(\Gamma_{l} \bar{J}_{p_{1}, k_{l}}+\bar{H}_{p_{1}, k_{l}}\right) E_{l}-\bar{E}_{l} H_{p_{1}, k_{m}}^{b}\right] \\
& -i k_{l}\left[\Delta_{l} J_{p_{1}, k_{p}}^{a}\left(\Gamma_{l} \bar{J}_{p_{2}, k_{l}}+\bar{H}_{p_{2}, k_{l}}\right)+\Sigma_{l} \bar{J}_{p_{2,} k_{l}}\left(\Gamma_{l} \bar{J}_{p_{1}, k_{l}}+H_{p_{1}, k_{l}}^{b}-A_{l} \Gamma_{l} H_{p_{1}, k_{l}}^{a}\right)\right. \\
& \left.+\Sigma_{l} \bar{H}_{p_{2}, k_{l}}\left(A_{l} \Gamma_{l} J_{p_{1}, k_{l}}^{b}-J_{p_{1}, k_{l}}^{a}+A_{l} \bar{H}_{p_{1}, k_{l}}\right)-\delta_{l} H_{p_{1}, k_{m}}^{b}\left(\bar{J}_{p_{2}, k_{l}}+A_{l} \bar{H}_{p_{2}, k_{l}}\right)\right] \\
& \left.+i k_{m} H_{p_{2}, k_{m}}^{b}\left[a_{l} J_{p_{1}, k_{p}}^{a}+f_{l}\left(\bar{J}_{p_{1}, k_{l}}+A_{l} \bar{H}_{p_{1}, k_{l}}\right)-\left(J_{p_{1}, k_{m}}^{b}+\bar{A}_{l} H_{p_{1}, k_{m}}^{b}\right)\right]\right\} \\
& \times\left(p_{1}^{2}-k_{m}^{2}\right)\left(p_{2}^{2}-k_{m}^{2}\right) .
\end{aligned}
$$

The variables $J$ and $H$ in the above equation are defined as

$$
\begin{aligned}
J_{p_{i}, p_{j}}^{r}= & {\left[p_{j} j_{l}\left(p_{i} r\right) j_{l}^{\prime}\left(p_{j} r\right)\right.} \\
& \left.-p_{i} j_{l}^{\prime}\left(p_{i} r\right) j_{l}\left(p_{j} r\right)\right] r^{2} /\left(p_{i}^{2}-p_{j}^{2}\right), \\
H_{p_{i}, p_{j}}^{r}= & {\left[p_{j} j_{l}\left(p_{i} r\right) h_{l}^{\prime}\left(p_{j} r\right)\right.} \\
& \left.\quad-p_{i} j_{l}^{\prime}\left(p_{i} r\right) h_{l}\left(p_{j} r\right)\right] r^{2} /\left(p_{i}^{2}-p_{j}^{2}\right), \\
\bar{J}_{p_{i} p_{j}}= & J_{p_{i}, p_{j}}^{b}-J_{p_{i}, p_{j}}^{a}, \\
\bar{H}_{p_{i}, p_{j}}= & H_{p_{i}, p_{j}}^{b}-H_{p_{i}, p_{j}}^{a},
\end{aligned}
$$

where $p_{i}, p_{j}$ equal $p_{1}, p_{2}, k_{p}, k_{l}$, and $k_{m} ; r$ equals $a$ and $b$. It is easy to show that as $b \rightarrow a$, or $k_{m} \rightarrow k_{l}$ and $\rho_{m} \rightarrow \rho_{l}$, our result is reduced to the case given in Ref. [3]. For the case of energy-shell scattering, $p_{1}=k_{m}$ and $p_{2}=k_{m}$, we get a simple expression of the scattering operator,

$$
T_{l}^{(s)}\left(k_{m}, k_{m}\right)=i \bar{A}_{l} / k_{m},
$$

which is the quantity used in the DOS calculation.

\section{APPENDIX B: SCATTERING AMPLITUDE FOR A SCATTERING UNIT CONSISTING OF $N$ SPHERES}

In this appendix we summarize the formalism involving a scattering unit consisting of $N$ solid spheres. The scattering unit is shown in Fig. 2(c). A plane acoustic wave of the form

$$
\psi=e^{i \mathbf{k}_{m} \cdot \mathbf{r}}
$$

is incident along the $z$ axis. The plane wave can be ex- panded in angular-momentum space as

$$
\psi=\sum_{l, m} 4 \pi i^{l} j_{l}\left(k_{m} r\right) Y_{l m}(\widehat{\mathbf{r}}) Y_{l m}^{*}\left(\widehat{\mathbf{k}}_{m}\right) \text {. }
$$

The expressions for the scalar potentials and displacements in each region are given by Eqs. (3.21) and (3.22) in Sec. III, with the summation $i$ extending from 1 to $N$.

When fitting boundary conditions at the $i_{0}$ th sphere surface, we transform all the scattered waves from other spheres $i \neq i_{0}$ into the form of spherical waves centered at sphere $i_{0}$ by using the transformation, Eq. (3.24),

$$
\begin{aligned}
\sum_{i\left(\neq i_{0}\right)} \sum_{l, m} Q_{l m}^{(i)} h_{l}\left(k_{l} r_{i}\right) Y_{l m}\left(\hat{\mathbf{r}}_{i}\right) & \\
=\sum_{i\left(\neq i_{0}\right)} \sum_{l^{\prime}, m^{\prime}} & {\left[\sum_{l, m} \sigma_{l^{\prime} m^{\prime}, l m}\left(\mathbf{d}_{i, i_{0}}\right) Q_{l m}^{(i)}\right] h_{l^{\prime}}\left(k_{l} r_{i_{0}}\right) } \\
& \times Y_{l^{\prime} m^{\prime}}\left(\hat{\mathbf{r}}_{i_{0}}\right),
\end{aligned}
$$

where $\mathrm{d}_{i, i_{0}}$ is the displacement vector from $O_{i}$ to $O_{i_{0}}$. Since there are three boundary conditions at the solidliquid interface, we can eliminate the three expansion coefficients $a_{l m}^{\left(i_{0}\right)}, b_{l m}^{\left(i_{0}\right)}$, and $c_{l m}^{\left(i_{0}\right)}$ in Eq. (3.22) to get an equation relating $Q_{l m}^{(i)}$ and $W_{l m}$,

$$
\begin{aligned}
& Q_{l m}^{\left(i_{0}\right)}+A_{l} \sum_{i\left(\neq i_{0}\right)} \sum_{l^{\prime}, m^{\prime}} Q_{l^{\prime} m^{\prime}}^{(i)} \sigma_{l m, l^{\prime} m^{\prime}}\left(\mathbf{d}_{i, i_{0}}\right) \\
& +A_{l} \sum_{l^{\prime}, m^{\prime}} W_{l^{\prime} m^{\prime}} s_{l m, l^{\prime} m^{\prime}}\left(\mathbf{d}_{i_{0}}\right)=0
\end{aligned}
$$

where $A_{l}$ is just the expansion coefficient of the scattered wave for a single solid sphere in liquid, and is given by Eq. (A6). Here $i_{0}=1,2, \ldots, N$.

The continuity of pressure and normal displacement at the scattering unit surface $S$ gives 


$$
\begin{array}{r}
\frac{\rho_{l}}{k_{l}} \sum_{l, m}\left\{\sum_{i} Q_{l m}^{(i)} \sum_{l^{\prime} m^{\prime}} s_{l^{\prime} m^{\prime}, l m}\left(-\mathbf{d}_{i}\right) h_{l^{\prime}}\left(k_{l} r\right) Y_{l^{\prime} m^{\prime}}(\hat{\mathbf{r}})+W_{l m} j_{l}\left(k_{l} r\right) Y_{l m}(\hat{\mathbf{r}})\right\} \\
=\left.\frac{\rho_{m}}{k_{m}} \sum_{l, m}\left\{Q_{l m}^{(M)} h_{l}\left(k_{m} r\right) Y_{l m}(\hat{\mathbf{r}})+4 \pi i^{l} j_{l}\left(k_{m} r\right) Y_{l m}^{*}\left(\hat{\mathbf{k}}_{m}\right) Y_{l m}(\hat{\mathbf{r}})\right\}\right|_{\mathbf{r} \text { on } S}, \\
\frac{1}{k_{l}} \frac{\partial}{\partial n}\left\{\sum_{l, m}\left[\sum_{i} Q_{l m}^{(i)} \sum_{l^{\prime} m^{\prime}} s_{l^{\prime} m^{\prime}, l m}\left(-\mathbf{d}_{i}\right) h_{l^{\prime}}\left(k_{l} r\right) Y_{l^{\prime} m^{\prime}}(\hat{\mathbf{r}})+W_{l m} j_{l}\left(k_{l} r\right) Y_{l m}(\hat{\mathbf{r}})\right]\right\} \\
=\left.\frac{1}{k_{m}} \frac{\partial}{\partial n}\left\{\sum_{l, m}\left[Q_{l m}^{(M)} h_{l}\left(k_{m} r\right) Y_{l m}(\hat{\mathbf{r}})+4 \pi i^{l} j_{l}\left(k_{m} r\right) Y_{l m}^{*}\left(\hat{\mathbf{k}}_{m}\right) Y_{l m}(\hat{\mathbf{r}})\right]\right\}\right|_{\mathbf{r} \text { on } S},
\end{array}
$$

where $n$ is the surface normal at $\mathbf{r}$. If the surface $S$ is not spherical in shape, we need to perform integrations over $S$ on both sides of the above two equations in order to get the expansion coefficients. This is the case for the two-sphere scattering unit. However, for a spherical $S$, Eq. (B5) becomes

$$
\begin{aligned}
& \frac{\rho_{l}}{k_{l}}\left\{\sum_{i, l^{\prime}, m^{\prime}} Q_{l^{\prime} m^{\prime}}^{(i)} s_{l m, l^{\prime} m^{\prime}}\left(-\mathbf{d}_{i}\right) h_{l}\left(k_{l} b\right)+W_{l m} j_{l}\left(k_{l} b\right)\right\}=\frac{\rho_{m}}{k_{m}}\left\{Q_{l m}^{(M)} h_{l}\left(k_{m} b\right)+4 \pi i^{l} j_{l}\left(k_{m} b\right) Y_{l m}^{*}\left(\hat{\mathbf{k}}_{m}\right)\right\}, \\
& \sum_{i, l^{\prime}, m^{\prime}} Q_{l^{\prime} m^{\prime}}^{(i)} s_{l m, l^{\prime} m^{\prime}}\left(-\mathbf{d}_{i}\right) h_{l}^{\prime}\left(k_{l} b\right)+W_{l m} j_{l}^{\prime}\left(k_{l} b\right)=Q_{l m}^{(M)} h_{l}^{\prime}\left(k_{m} b\right)+4 \pi i^{l} j_{l}^{\prime}\left(k_{m} b\right) Y_{l m}^{*}\left(\hat{\mathbf{k}}_{m}\right) .
\end{aligned}
$$

$W_{l m}$ and $Q_{l m}^{(M)}$ may be eliminated from Eqs. (B5c) and (B5d) to get the equation satisfied by $Q_{l m}^{(i)}$ as

$$
\begin{array}{r}
\frac{1}{A_{l}} Q_{l m}^{\left(i_{0}\right)}+\sum_{i\left(\neq i_{0}\right)} \sum_{l^{\prime}, m^{\prime}} \sigma_{l m, l^{\prime} m^{\prime}}\left(\mathbf{d}_{i, i_{0}}\right) Q_{l^{\prime} m^{\prime}}^{(i)}-\sum_{i} \sum_{l^{\prime}, m^{\prime}}\left[\sum_{l^{\prime \prime}, m^{\prime \prime}} s_{l m, l^{\prime \prime} m^{\prime \prime}}\left(\mathbf{d}_{i_{0}}\right) t_{\left.l^{\prime \prime} s_{l^{\prime \prime} m^{\prime \prime}, l^{\prime} m^{\prime}}\left(-\mathbf{d}_{i}\right)\right] Q_{l^{\prime} m^{\prime}}^{(i)}}=\sum_{l^{\prime}, m^{\prime}} 4 \pi i^{l^{\prime}} t_{l^{\prime}}^{I} S_{l m, l^{\prime} m^{\prime}}\left(\mathbf{d}_{i_{0}}\right) Y_{l^{\prime} m^{\prime}}^{*}\left(\hat{\mathbf{k}}_{m}\right),\right.
\end{array}
$$

where

$$
\begin{aligned}
& t_{l}^{Q}=\left.\frac{k_{l} \rho_{m} h_{f}^{\prime} h_{m}-k_{m} \rho_{l} h_{f} h_{m}^{\prime}}{k_{l} \rho_{m} j_{f}^{\prime} h_{m}-k_{m} \rho_{l} j_{f} h_{m}^{\prime}}\right|_{r=b}, \\
& t_{l}^{I}=\left.i \frac{k_{l} \rho_{m} / x_{m}^{2}}{k_{l} \rho_{m} j_{f}^{\prime} h_{m}-k_{m} \rho_{l} j_{f} h_{m}^{\prime}}\right|_{r=b} .
\end{aligned}
$$

After getting $Q_{l m}^{(i)}$, we can obtain $Q_{l m}^{(M)}$ as

$$
Q_{l m}^{(M)}=\bar{t}_{l}^{Q} \sum_{i} \sum_{l^{\prime}, m^{\prime}} s_{l m, l^{\prime} m^{\prime}}\left(-\mathbf{d}_{i}\right) Q_{l^{\prime} m^{\prime}}^{(i)}-4 \pi i{ }^{l} \bar{t}_{l}^{I} Y_{l m}^{*}\left(\hat{\mathbf{k}}_{m}\right)
$$

Here $\bar{t}_{l}^{Q}$ and $\bar{t}_{l}^{I}$ are defined as

$$
\begin{aligned}
& \bar{t}_{l}^{Q}=\frac{k_{m} \rho_{l}}{k_{l} \rho_{m}}\left[h_{f}-t_{l} Q_{j}\right] /\left.h_{m}\right|_{r=b}, \\
& \bar{t}_{l}^{I}=\left[\frac{k_{m} \rho_{l}}{k_{l} \rho_{m}} t_{l}^{I} j_{f}+j_{m}\right] /\left.h_{m}\right|_{r=b} .
\end{aligned}
$$

If one denotes the coefficient matrix of the linear Eq. (B6) by $M_{i_{1} l_{1} m_{1}, i_{2} l_{2} m_{2}}$ and the corresponding components for the inverse of the coefficient matrix $M$ by $M_{i_{1} l_{1} m_{1}, i_{2} l_{2} m_{2}}^{-1}$, then

$$
M_{i_{1} l_{1} m_{1}, i_{2} l_{2} m_{2}}=\frac{1}{A_{l}} \delta_{i_{1}, i_{2}} \delta_{l_{1}, l_{2}} \delta_{m_{1}, m_{2}}+\left.\sigma_{l_{1} m_{1}, l_{2} m_{2}}\left(\mathbf{d}_{i_{2}, i_{1}}\right)\right|_{i_{1} \neq i_{2}}-\sum_{l^{\prime}, m^{\prime}} s_{l_{1} m_{1}, l^{\prime} m^{\prime}}\left(\mathbf{d}_{i_{1}}\right) t_{l^{\prime}} s_{l^{\prime} m^{\prime}, l_{2} m_{2}}\left(-\mathbf{d}_{i_{2}}\right)
$$

By defining

$$
\Pi_{l m, l^{\prime} m^{\prime}}=\bar{t}_{l}^{I} \delta_{l, l^{\prime}} \delta_{m, m^{\prime}}-(-i)^{l} \bar{t}_{l}^{Q} \bar{M}_{l m, l^{\prime} m^{\prime}} i^{l^{\prime}} t_{l^{\prime}}^{I}
$$

where $\bar{M}_{l m, l^{\prime} m^{\prime}}$ is given by

$$
\bar{M}_{l m, l^{\prime} m^{\prime}}=\sum_{i_{1}, i_{2}} \sum_{l_{1}, m_{1}} \sum_{l_{2}, m_{2}} s_{l m, l_{1} m_{1}}\left(-\mathbf{d}_{i_{1}}\right) \boldsymbol{M}_{i_{1} l_{1} m_{1}, i_{2} l_{2} m_{2}}^{-1} s_{l_{2} m_{2}, l^{\prime} m^{\prime}}\left(\mathbf{d}_{i_{2}}\right)
$$


then the scattering amplitude from $\mathbf{k}_{m}$ to $\mathbf{k}_{m}^{\prime}\left(\left|\mathbf{k}_{m}^{\prime}\right|=\left|\mathbf{k}_{m}\right|=k_{m}\right)$ is given by

$$
f_{\mathbf{k}_{m}^{\prime}, \mathbf{k}_{m}}=\frac{4 \pi i}{k_{m}} \sum_{l, m} \sum_{l^{\prime}, m^{\prime}} Y_{l m}\left(\hat{\mathbf{k}}_{m}^{\prime}\right) \Pi_{l m, l^{\prime} m^{\prime}} Y_{l^{\prime} m^{\prime}}^{*}\left(\hat{\mathbf{k}}_{m}\right)
$$

Taking the average over all the incident wave directions yields

$$
f\left(k_{m}\right)=\int f_{\mathbf{k}_{m}, \mathbf{k}_{m}} d \Omega_{\mathbf{k}_{m}} / 4 \pi=\frac{i}{k_{m}} \sum_{l, m} \Pi_{l m, l m} .
$$

If the effective medium is the same as the fluid inside the scattering unit, then our result, Eq. (B13), reduces to that for the scattering of a cluster of spheres immersed in fluid. It is also easy to generalize our result to the case where the spheres are not identical. This can be done by setting the single-sphere scattering expansion coefficient, $A_{l}$ in Eq. (B.6), to equal that for the particular sphere.

The displacement transformation matrix $s_{l^{\prime} m^{\prime}, l m}(\mathbf{D})$ in Eq. (3.24) is given by $[17,18]$

$$
\begin{aligned}
& s_{l^{\prime} m^{\prime}, l m}(\mathbf{D})=\sum_{\lambda}(-1)^{\left(l^{\prime}-l+\lambda\right) / 2}(-1)^{m}\left[4 \pi\left(2 l^{\prime}+1\right)(2 l+1)(2 \lambda+1)\right]^{1 / 2} \\
& \times\left(\begin{array}{lll}
l^{\prime} & l & \lambda \\
0 & 0 & 0
\end{array}\right)\left(\begin{array}{ccc}
l^{\prime} & l & \lambda \\
m^{\prime} & -m & m-m^{\prime}
\end{array}\right] j_{\lambda}(k D) Y_{\lambda m-m^{\prime}}(\hat{\mathbf{D}}) .
\end{aligned}
$$

Here ( ) represents the 3-j symbol. The expression for $\sigma_{l^{\prime} m^{\prime}, l m}(\mathbf{D})$ is the same as the above equation except $j_{\lambda}(k D)$ is replaced by $h_{\lambda}(k D)$.

*Present address: Department of Chemical Engineering and Materials Science, University of Minnesota, Minneapolis, MN 55455.

[1] M. A. Biot, J. Acoust. Soc. Am. 28, 168 (1956); 28, 5848 (1956).

[2] T. J. Plona, Appl. Phys. Lett. 36, 259 (1980).

[3] L. Schwartz and T. J. Plona, J. Appl. Phys. 55, 3971 (1984).

[4] L. Schwartz and D. L. Johnson, Phys. Rev. B 30, 4302 (1984).

[5] J. J. Faran, Jr., J. Acoust. Soc. Am. 23, 405 (1951).

[6] J. G. Fikioris and P. C. Waterman, J. Math. Phys. 5, 1413 (1964).

[7] P. C. Waterman, J. Acoust. Soc. Am. 45, 1417 (1969).

[8] M. Lax, Rev. Mod. Phys. 23, 287 (1951); L. Tsang and J. A. Kong, J. Appl. Phys. 51, 3465 (1980); 52, 5448 (1981).

[9] L. Tsang, J. A. Kong, and T. Habashy, J. Acoust. Soc. Am. 71, 552 (1982).

[10] A. J. Devaney, J. Math. Phys. 21, 2603 (1980).

[11] See Scattering and Localization of Classical Waves in Random Media, edited by P. Sheng (World Scientfic, Singapore, 1990).

[12] J. Liu, L. Ye, D. A. Weitz, and P. Sheng, Phys. Rev. Lett. 65, 2602 (1990).

[13] X. Jing, P. Sheng, and M. Zhou, Phys. Rev. Lett. 66, 1240 (1991).
[14] See, for eample, L. M. Brekhovskikh, Wave in Layered Media (Academic, New York, 1980).

[15] See E. N. Economou, Green's Function in Quantum Physics, 2nd ed. (Springer-Verlag, New York, 1983), p. 141.

[16] See, for example, P. M. Morse and H. Feshbach, Method of Theoretical Physics (McGraw-Hill, New York, 1953), p. 1898.

[17] M. Danos and L. C. Maximon, J. Math. Phys. 6, 766 (1965).

[18] B. Peterson and S. Ström, Phys. Rev. D 8, 3661 (1973).

[19] G. J. Throop and R. J. Bearman, J. Chem. Phys. 42, 2408 (1965).

[20] C. F. Bohren and D. R. Huffman, Absorption and Scattering of Light by Small Particles (Wiley-Interscience, New York, 1983), p. 325.

[21] J. Virieux, Geophys. 51, 889 (1986).

[22] By expressing all th solid and fluid euqations as a system of coupled first-order partial-differential equations, it can be shown that the direct calculation of finite differences across interfaces guarantees the boundary conditions. See B. Fornberg, Geophys. 52, 483 (1987).

[23] J. C. Maxwell-Garnett, Philos. Trans. R. Soc. 203, 385 (1904).

[24] See, for example, M. Born and E. Wolf, Principle of Optics, 2nd ed. (Macmillan, New York, 1964), p. 633.

[25] P. Sheng, Phys. Rev. Lett. 45, 60 (1980). 


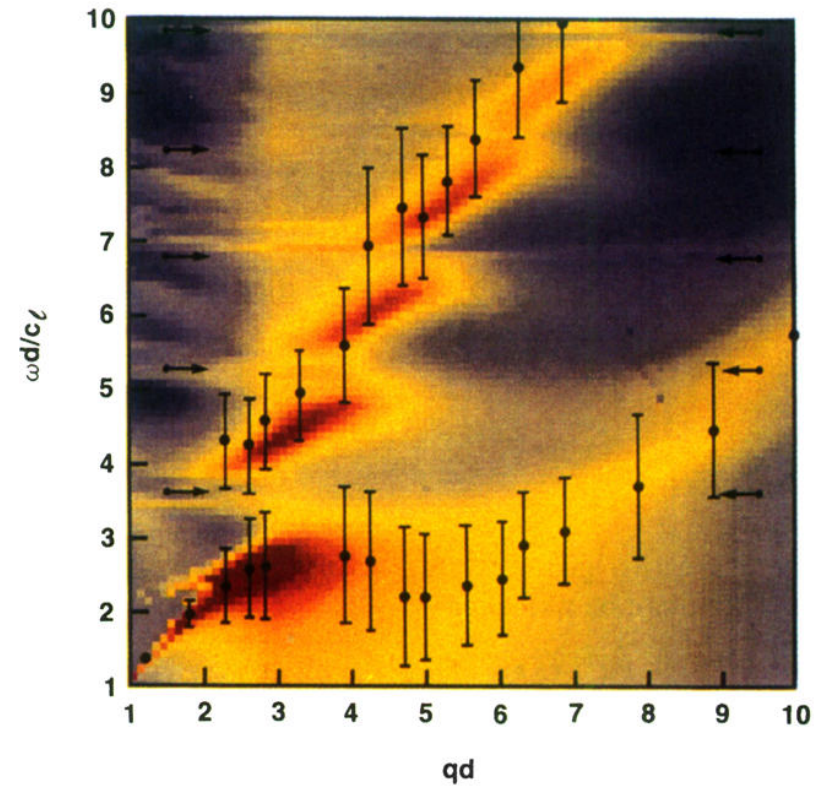

FIG. 11. The DOS for a two-sphere scattering unit embedded in an effective medium with $\Phi=0.51$. This figure should be compared with Fig. 3(b). 


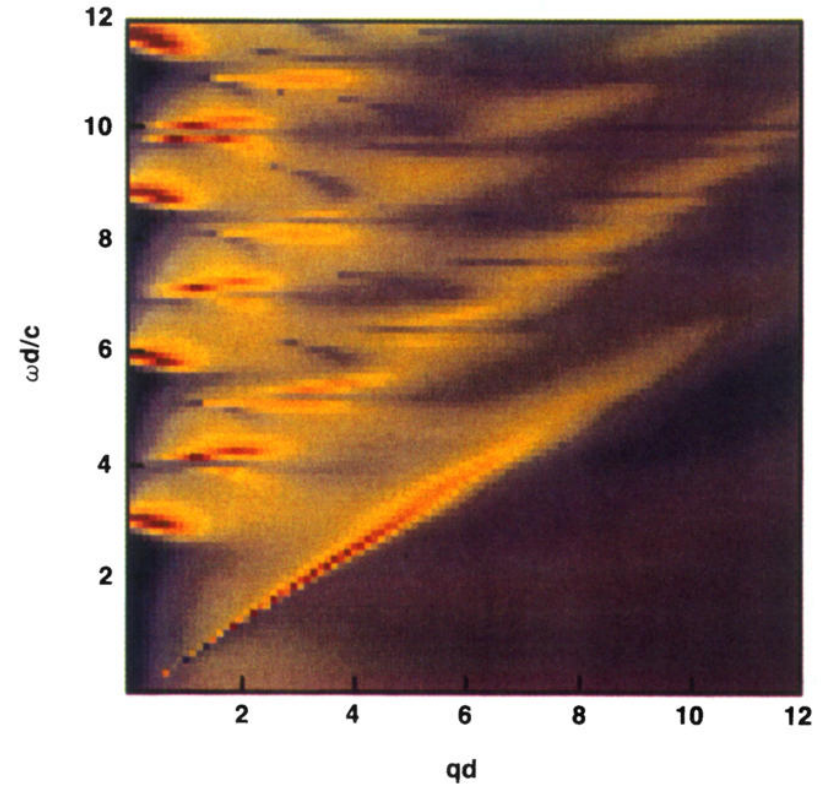

FIG. 19. The DOS of a single-coated dielectric sphere in effective medium, plotted as a function of reduced wave vector $q d$ and reduced frequency $\omega d / c$. The color scheme is the same as that of Fig. 3. The dielectric constants of the sphere and the matrix are $\epsilon_{s}=4.0$ and $\epsilon_{0}=1.0$, respectively, and $\Phi=0.3$. 

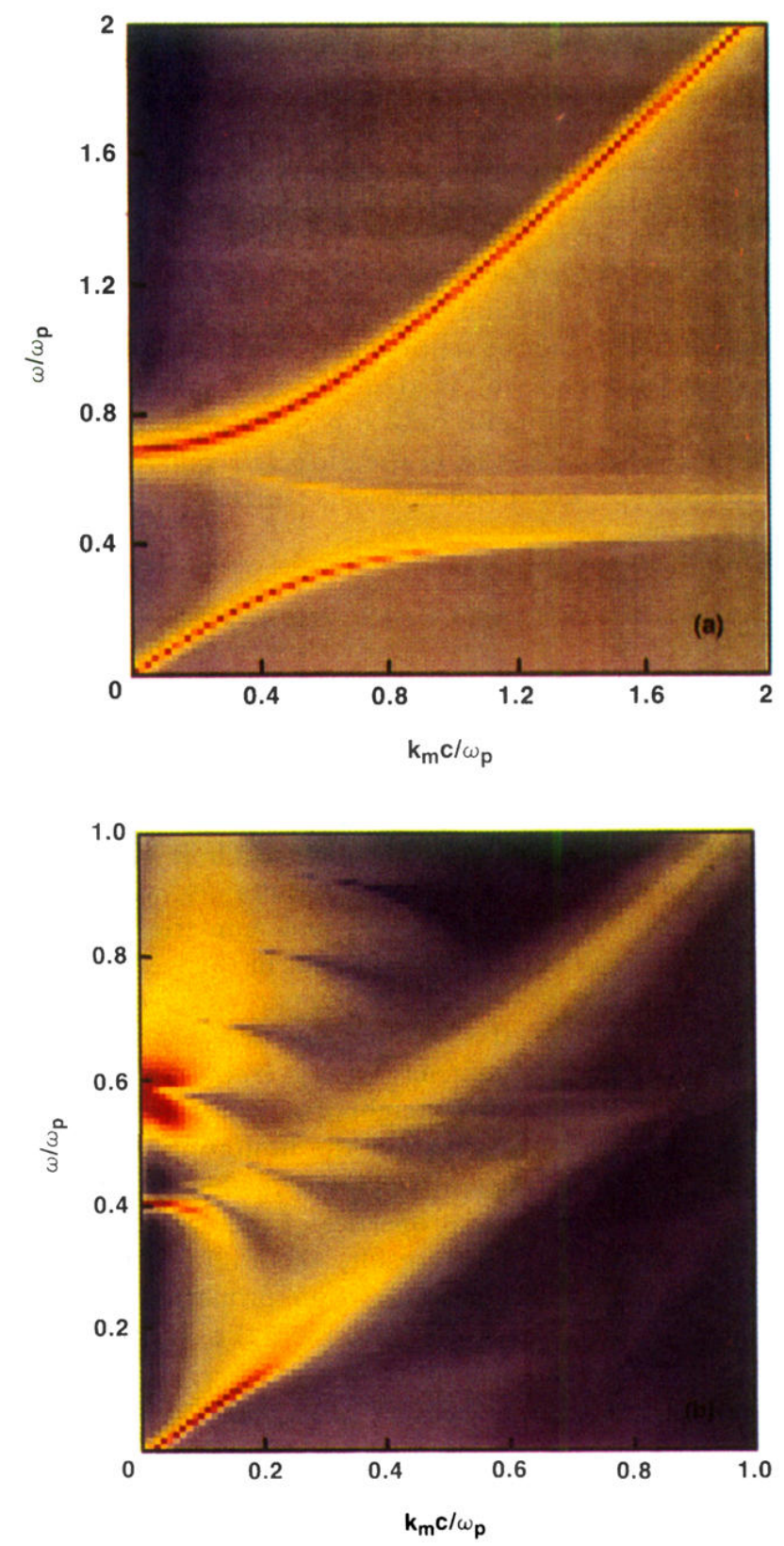

FIG. 20. The DOS for a single-coated metallic sphere in effective medium with $\Phi=0.3$. The color scheme is the same as that of Fig. 3. (a) The reduced sphere size is $\omega_{p} d / c=2.0$. (b) The reduced sphere size is $\omega_{p} d / c=12.0$. 

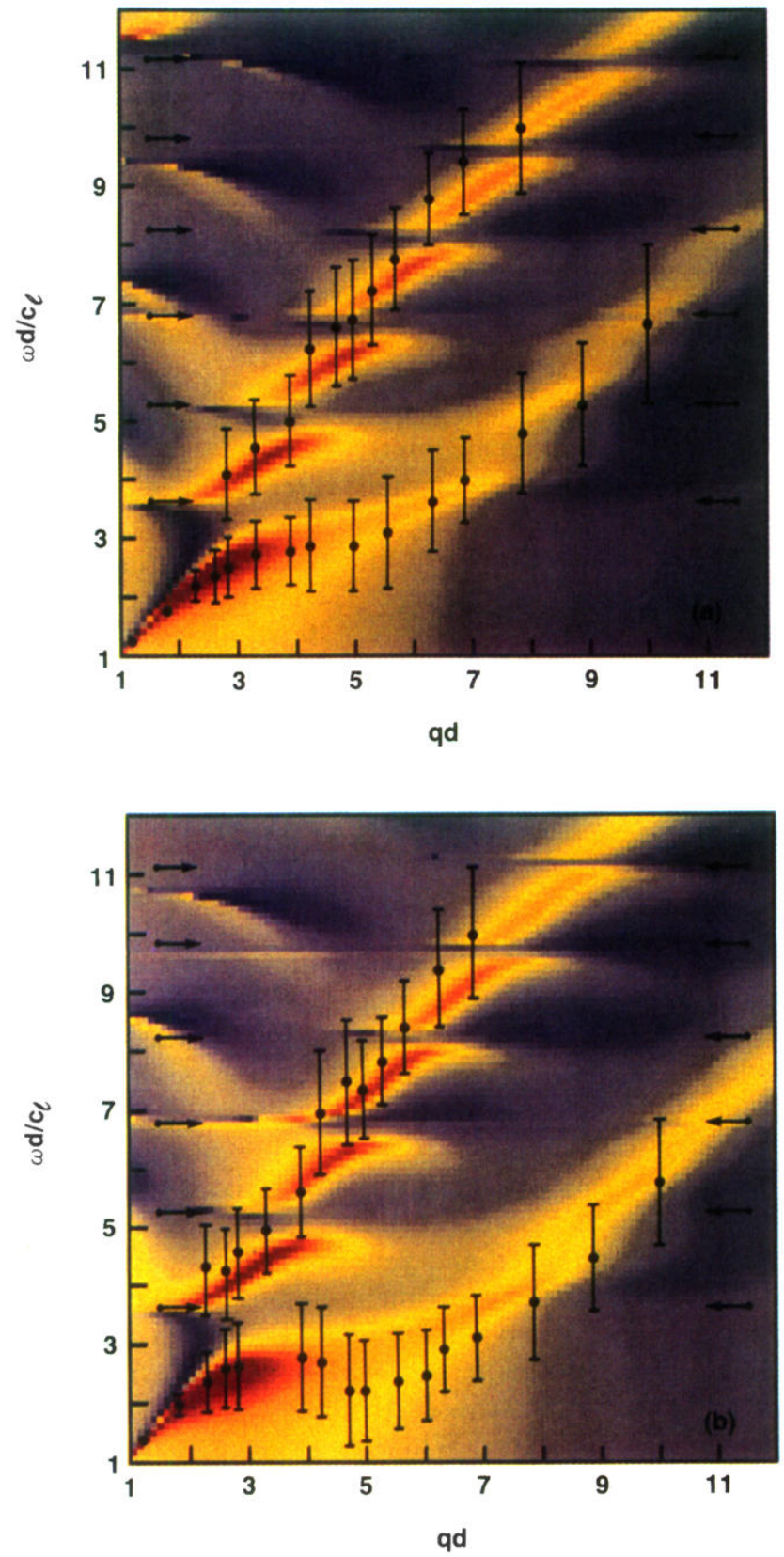

FIG. 3. The DOS plotted as a function of $\omega d / c_{l}$ and $q d$. The magnitude of the DOS is delineated by color, where high is indicated by red and low is indicated by blue. Yellow is intermediate. Experimental data are shown as solid circles. The bars indicate their frequency widths. The arrows on the sides indicate the frequency positions of the scattering cross-section peaks for a single sphere immersed in liquid, shown in Fig.4. (a) $\Phi=0.38$ and (b) $\Phi=0.51$. 